\title{
Multiple Risky Assets, Transaction Costs and Return Predictability: Implications for Portfolio Choice*
}

\author{
Anthony W. Lynch ${ }^{\dagger}$ \\ New York University and NBER \\ Sinan $\operatorname{Tan}^{\ddagger}$ \\ New York University \\ First Version: 15 November 2002 \\ This Version: 17 December 2002 \\ Work in progress. \\ Comments welcome.
}

\footnotetext{
*The authors would like to thank Hong Liu, Steve Figlewski and seminar participants at NYU for their helpful comments. All remaining errors are of course the authors' responsibility.

${ }^{\dagger}$ Stern School of Business, New York University, 44 West Fourth Street, Suite 9-190, New York, NY 100121126, alynch@stern.nyu.edu, (212) 998-0350.

${ }^{\ddagger}$ Stern School of Business, New York University, 44 West Fourth Street, Suite 9-190, New York, NY 100121126, stan@stern.nyu.edu, (212) 998-0560.
} 


\title{
Multiple Risky Assets, Transaction Costs and Return Predictability: Implications for Portfolio Choice
}

\begin{abstract}
Our paper contributes to the dynamic portfolio choice and transaction cost literatures by considering a multiperiod CRRA individual who faces transaction costs and who has access to multiple risky assets, all with predictable returns. We numerically solve the individual's multiperiod problem in the presence of transaction costs and predictability. In particular, we characterize the investor's optimal portfolio choice with proportional and fixed transaction costs, and with return predictability similar to that observed for the U.S. stock market. We also perform some comparative statics to better understand the nature of the no-trade region with more than one risky asset. Throughout our focus is on the case with two risky assets. We also perform some utility comparisons. The calibration exercise reveals some interesting results about the relative attractiveness of the three equity portfolios calibrated.

With proportional transaction costs and i.i.d. returns, we numerically find the rebalancing rule to be a no-trade region for the portfolio weights with rebalancing to the boundary. With zero correlation, the no-trade region is a rectangle irrespective of the investor's age. When the correlation of the risky assets is non-zero, the no-trade region becomes a parallelogram. With positive correlation, the parallelogram distorts the associated rectangle in such a way as to take advantage of the associated substitutability across the two assets that the positive correlation induces. The converse is true for negative correlation. Turning to the allocations with return predictability, our numerical results strongly suggest that it is the conditional return correlation that determines the nature of the distortion to the no-trade parallelogram. Irrespective of the investor's age, the distortion always mirrors the no-trade parallelogram distortion that we find in the i.i.d. case for return correlation of the same sign. The no-trade region is always larger late in life than early in life. However, the difference in no-trade area between early and late in life is less pronounced when returns are predictable, consistent with intuition that the benefits from rebalancing today are more short-lived when returns are predictable than in the i.i.d. case.
\end{abstract}




\section{Introduction}

Dynamic portfolio choice has received considerable attention in recent years, spurred by evidence that returns are predictable. ${ }^{1}$ Most of the papers consider the case with a single risky asset, but exceptions include Campbell, Chan and Viceira (2001) and Lynch (2001). ${ }^{2}$ Both these papers examine an investor who has access to multiple risky assets whose returns are predictable. While portfolio rebalancing typically requires the payment of transaction costs, this is a complication that has been largely ignored by this literature, though papers by Balduzzi and Lynch (1998) and Lynch and Balduzzi (2000)consider their effect when one predictable risky asset return is available. In addition, a large literature has considered the effects of transaction costs on portfolio choices, but all the papers assume that the opportunity set is constant through time. A very small number of these papers allow access to more than one risky asset, most notably Leland (2000) and Liu (2002).

Our paper contributes to the dynamic portfolio choice and transaction cost literatures by considering a multiperiod individual who faces transaction costs and who has access to multiple risky assets, all with predictable returns. We numerically solve the individual's multiperiod problem in the presence of transaction costs and predictability. In particular, we characterize the investor's optimal portfolio choice with proportional transaction costs, and with return predictability similar to that observed for the U.S. stock market. We also perform some comparative statics to better understand the nature of the no-trade region with more than one risky asset. Throughout our focus is on the case with two risky assets.

We also perform some utility comparisons. With these calculations, we are interested in the fraction of wealth that an investor who is using a given policy, would be prepared to give up to be allowed to use an alternate policy. Four utility comparisons are performed. First, the utility cost of transaction costs are assessed. Second, the utility cost of holding one risky asset rather than another risky asset is assessed for all possible pairwise combinations of the risky asset portfolios.

\footnotetext{
${ }^{1}$ Campbell (1987) and Fama and French (1989), among others, find that stock return variation can be explained by the one-month Treasury bill rate, by a contemporaneous and a lagged measure of the term premium, and by the dividend yield.

${ }^{2}$ Brennan and Schwartz (1996) and Brennan, Schwartz, and Lagnado (1996) analyze numerically the impact of myopic versus dynamic decision-making when the investor bases decisions on the conditional distribution of returns. Campbell and Viceira (1996) obtain a closed-form solution to the investor's multiperiod problem in the presence of predictability by using log-linear approximations to the budget constraint. Kim and Omberg (1996) solve the continuous-time analog without any approximations. Barberis (1996) considers the effect of asset-return predictability on myopic portfolio choices at different investment horizons; he also considers the effect of predictability on dynamic portfolio choices, when the investor rebalances every year. Finally, Kandel and Stambaugh (1996) explore the effects of predictability in a myopic setting, where the investor rebalances monthly. These last two papers account for the effects of estimation error on portfolio choice.
} 
Third, the utility cost of holding one or other of the two risky assets rather than both is assessed. All three of these comparisons are performed with and without predictability. Finally, the utility cost of ignoring predictability is assessed for cases in which the investor has access to one or both risky assets. That is, in a world with predictability, we are interested in the fraction of wealth that an investor who is using the i.i.d. policy, would be prepared to give up to be allowed to use the optimal policy with predictability.

All the utility comparisons and many of the optimal allocations are obtained for risky assets whose returns are calibrated to portfolios of U.S. stocks. The three portfolios are the value-weighted market, a low book-to-market (B-M) portfolio and a high B-M portfolio. Return predictability is calibrated to that of dividend yield over the period from $7 / 27$ to 11/96. Transaction cost parameters are chosen to mirror real world trading costs faced by investors trading mutual funds with the characteristics of the three stock portfolios considered.

Transaction costs can be modelled either as proportional to the change in the holding of the risky asset, or as a fixed fraction of portfolio value. With one risky asset and a constant opportunity set, Constantinides (1986) finds that proportional transaction costs affect portfolio choice since the optimal policy is a no-trade region with return to the closer boundary when rebalancing. Davis and Norman (1990) consider the same problem, and are able to solve it exactly, without imposing restrictions on the consumption process. Morton and Pliska (1993) and Schroder (1995) characterize portfolio choice with fixed transaction costs and find that the optimal policy is a no-trade region with a single return point inside the region. All of these papers allow continuous decision-making and impose a constant opportunity set. ${ }^{3}$ With more than one risky asset, the proportional cost parameter can differ across assets and there are two types of fixed cost that the investor can face. One type is a single fixed cost that is incurred if either asset is rebalanced. The other specifies a fixed cost for each risky asset which is triggered by any rebalancing of that asset. We consider both types of fixed costs.

Our paper is most closely related to recent work by Akian, Menaldi and Sulem (1996), Leland (2000) and Liu (2002) who all consider portfolio choice problems with multiple risky assets and

\footnotetext{
${ }^{3}$ Several recent papers account for transaction costs in general equilibrium models, in empirical testing, and with different decision-making structures. Koo (1991) and Vayanos (1996) investigate the equilibrium implications of proportional transaction costs. Heaton and Lucas (1996) consider a dynamic equilibrium model where infinitely-lived workers trade a bond and a stock, and show that large transaction costs are needed to generate a sizable equity premium in equilibrium. Hansen, Heaton, and Luttmer (1995), Luttmer (1996a), and He and Modest (1995) develop pricing-operator tests of asset-pricing models which explicitly account for bid-asked spreads and short-sale constraints. Duffie and Sun (1990) model investors who face fixed transaction costs and decide the interval of time until their next rebalancing at the time of their current rebalancing. The authors find that the optimal interval between trades is a constant.
} 
transaction costs. But in contrast to our paper, the opportunity set is constant in all these papers. Leland examines an investor who cares about maintaining a target portfolio weight for each stock. The investor's objective is to minimize the sum of the proportional transaction costs and the variance of the tracking error. Leland develops a numerical procedure to calculate the optimal rebalancing rule and then implements the procedure for a number of examples. Liu considers a CARA investor facing uncorrelated risky asset returns and is able to obtains a solution that is almost closed-form for fixed and proportional costs. In one section, he briefly considers the case of non-zero correlation but in doing so, assumes that the boundaries of the no-trade region are straight lines. Aakian, Menaldi and Sulem (1996) consider a CRRA investor facing proportional costs to trade assets whose prices follow geometric Brownian motion. They prove existence and uniqueness of a solution and then present some numerical results for the one- and two-risky-asset cases.

With proportional transaction costs and i.i.d. returns, we numerically find the rebalancing rule to be a no-trade region for the portfolio weights whose shape depends on the correlation between the two risky assets. With zero correlation, the no-trade region is a rectangle irrespective of the investor's age. This result is consistent with Liu's finding of a no-trade rectangle for dollar holdings when the investor has exponential utility and the two returns exhibit zero correlation. When the correlation of the risky assets is non-zero, the no-trade region becomes a parallelogram, which suggests that a similar finding by Leland is robust to using a utility-based objective function for the investor. With positive correlation, the parallelogram distorts the associated rectangle in such a way as to take advantage of the associated substitutability across the two assets that the positive correlation induces. The converse is true for negative correlation.

Turning to the allocations with return predictability, we consider three cases, one that matches the conditional correlation to the data, another that sets the conditional correlation to zero in all states, and a third that allows the conditional correlation to vary across states. For the pair of risky assets considered, the data exhibits positive correlation and, as in the i.i.d. case, the no-trade region is a parallelogram, but now there is a no-trade region for each state. As before, the no-trade parallelograms distort the associated rectangles to take advantage of the substitutability of the two risky assets.

Our numerical results strongly suggest that it is the conditional return correlation that determines the nature of the distortion to the no-trade parallelogram. For the case with the conditional correlation set to zero in all states, the no-trade region is always a rectangle, which is the same 
shape as the no-trade region in the i.i.d. case with zero correlation. For the case with conditional correlation that varies by state, the distortion to the no-trade parallelogram varies depends on the conditional return correlation in that state. Irrespective of the investor's age, the distortion always mirrors the no-trade parallelogram distortion that we find in the i.i.d. case for return correlation of the same sign. This finding that the no-trade region state by state mirrors its shape in the i.i.d. case is consistent with numerical results in Balduzzi and Lynch (1999) for the case with one risky asset.

We also examine how predictability affects the size or area of the no-trade region. The benefits of rebalancing must be zero after the terminal date and so are smaller late than early in life (see Balduzzi and Lynch, 1999). Consequently, the no-trade region is always larger late in life than early in life. However, the difference in no-trade area between early and late in life is less pronounced when returns are predictable, consistent with intuition that the benefits from rebalancing today are more short-lived when returns are predictable than in the i.i.d. case. We also examine what happens to the portfolio rebalancing rule in the presence of fixed as well as proportional costs.

Turning to the utility cost results, we find the utility cost of having access to only one of two risky assets is always higher when returns are predictable rather than i.i.d, even though the utility cost of having access to only one asset and not another can sometimes be higher when returns are i.i.d. rather than predictable. Further, this utility cost can either increase or decrease with the introduction of proportional costs. Since higher return volatility causes transaction costs to inflict a greater utility loss, the direction most likely depends on the return volatility for the lone asset as compared with the return volatility for the optimal portfolio of the two assets. Finally, the utility cost of ignoring return predictability when the investor has access to two assets always lies between the values for this cost when the investor has access to one or other of the assets alone.

The calibration exercise reveals some interesting results about the relative attractiveness of the three equity portfolios calibrated. When an investor with access to the market is given access to the high B-M portfolio, the investor chooses to always hold or almost always hold only the high B-M portfolio depending on whether returns are i.i.d. or predictable, respectively. This result is robust to the introduction of proportional costs, even though the proportional cost for the high B-M portfolio is realistically chosen to be higher than that for the market. Return predictability increases the high B-M portfolio's attractiveness relative to the market, with the utility cost of holding the market and not the high B-M portfolio going from around $6 \%$ of wealth with i.i.d. returns to over $13 \%$ with predictability. When the low B-M portfolio is made available in addition 
to the market, the investor only holds it when returns are predictable. When returns are i.i.d., the investor continues to hold only the market. The utility cost of not having access to the low B-M portfolio as well as the market is always less than $1 \%$ of wealth.

The paper is organized as follows. Section 2 describes the investor's dynamic optimization problem with predictable returns and transaction costs. Section 3 calibrates the state variables and asset returns to the U.S. economy. Section 4 discusses the portfolio rebalancing rules, while Section 5 performs utility comparisons across different investor problems. Section 6 concludes.

\section{The investor problem}

This section lays out the preferences of and constraints faced by the investor. We characterize the optimization problem for a dynamic investor who does or does not take into account asset-return predictability. We also describe the nature of the utility comparisons to be performed later in the paper, and the solution technique for numerically solving the investor's problem.

\subsection{Constraints and preferences}

We consider the portfolio allocation between $N$ risky assets and a riskless asset. The investor faces transaction costs that are proportional to wealth. The law of motion of the investor's wealth, $W$, is given by

$$
W_{t+1}=\left(W_{t}-c_{t}\right)\left(1-f_{t}\right)\left[\alpha_{\mathbf{t}}^{\prime}\left(\mathbf{R}_{\mathbf{t}+\mathbf{1}}-R_{t}^{f} \mathbf{i}_{\mathbf{N}}\right)+R_{t}^{f}\right]
$$

where $c$ is consumption, $\alpha$ is an $N x 1$ vector of portfolio weights in the $N$ risky assets, $\mathbf{R}$ is an $N x 1$ vector of returns on the $N$ risky assets, $R^{f}$ is the risk-free rate, and $f$ is the transactions cost per dollar of portfolio value. We further define $\kappa$ as the fraction of wealth consumed and $R_{W}$ as the rate of return on wealth, net of the transaction costs incurred. Hence, we have

$$
W_{t+1}=\left(1-\kappa_{t}\right) W_{t} R_{W, t+1}
$$

The law of motion for wealth in eq. (1) implicitly assumes that consumption at time $t$ and any transaction costs to be paid at time $t$ are obtained by liquidating costlessly the $i$ th risky asset and the riskless asset in the proportions $\hat{\alpha}^{i}$ and $\left(1-\hat{\alpha}^{\prime} \mathbf{i}_{\mathbf{N}}\right)$, where $\hat{\alpha}^{i}$ is the allocation to the $i$ th risky asset inherited from the previous period:

$$
\hat{\alpha}_{t+1}^{i} \equiv \frac{\alpha_{t}^{i}\left(1-\kappa_{t}\right) W_{t}\left(1-f_{t}\right) R_{t+1}^{i}}{W_{t+1}}=\frac{\alpha_{t}^{i} R_{t+1}^{i}}{\alpha_{\mathbf{t}}^{\prime}\left(\mathbf{R}_{\mathbf{t}+\mathbf{1}}-R_{t}^{f} \mathbf{i}_{\mathbf{N}}\right)+R_{t}^{f}} .
$$


and $\hat{\alpha}$ is the $N x 1$ vector of these inherited portfolio weights. This assumption is not so onerous given the availability of money-market bank accounts and given that equities pay dividends. To the extent that the sum of the risky assets' dividends exceeds the consumption out of the risky asset, $\kappa\left(\hat{\alpha}^{\prime} \mathbf{i}_{\mathbf{N}} \mathbf{W}\right.$, a dividend reinvestment plan can be used to costlessly reinvest the excess dividend in the risky asset.

In general, we model the cost of transacting, $f$, as a function of the difference between the $N x 1$ vector of end-of-period wealth allocations to the risky assets, $\hat{\alpha}$, and $\alpha:^{4}$

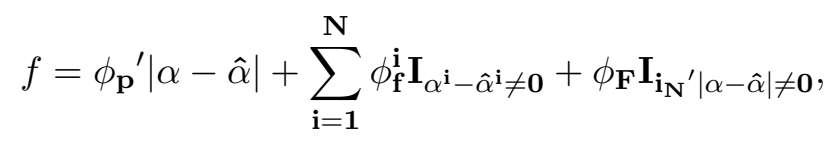

where $I_{y \neq 0}$ is an indicator function which equals one if $y \neq 0$ and zero otherwise. Specifically, we assume transaction costs to have three components. The first is the proportional cost which for each asset is proportional to the change in the value of the risky asset holding, as in Constantinides (1986). The $N \mathrm{x} 1$ vector $\phi_{\mathbf{p}}$ has $i$ th element $\phi_{p}^{i}$ which gives the proportional cost associated with trading the $i$ th risky asset. The last two terms are fixed fraction of the total value of the portfolio, as in Morton and Pliska (1993) and Schroder (1995). Both reflect fixed costs of rebalancing the portfolio that are the same regardless of the size of the rebalancing. These fixed costs are allowed to increase with the investor's wealth, since they are likely to depend on the opportunity cost of the investor's time. We consider two such costs. With the first, a separate fixed cost is associated with each risky asset. If both risky assets are rebalanced, both fixed costs are incurred, but if only one is rebalanced, only the fixed cost associated with that asset is incurred. The $N x 1$ vector $\phi_{\mathbf{f}}$ has $i$ th element $\phi_{f}^{i}$ which gives the fixed cost triggered by any trading of the $i$ th risky asset. With the last term, the single fixed cost $\phi_{F}$ is incurred if at least one risky asset is rebalanced.

We allow returns to be predictable and assume that there exists a "predictive" variable, $D$ (to be explicitly identified later in the calibration exercise) which affects the conditional mean of the risky assets' return. We assume $D$ follows a first-order Markov process. For simplicity, the riskless rate is assumed to be constant, and so $R_{t}^{f}=R^{f}$ for every t.

We consider the optimal portfolio problem of a investor with a finite life of $T$ periods and utility over intermediate consumption. Preferences are time separable and exhibit constant relative risk aversion (CRRA):

$$
E\left[\sum_{t=1}^{T} \delta^{t} \frac{c_{t}^{1-\gamma}}{1-\gamma} \mid D_{1}, \hat{\alpha}_{1}\right]
$$

\footnotetext{
${ }^{4}$ To keep notation simple, we drop the time subscript when all variables in a mathematical expression are contemporaneous.
} 
where $\gamma$ is the relative-risk-aversion coefficient and $\delta$ is the time-discount parameter. Note that the expected lifetime utility depends on the state of the economy at time 1. Further, the inherited

portfolio weight for the $i$ th risky asset $\hat{\alpha}_{1}^{i}$ is a state variable when at least one of $\phi_{p}^{i}$, $\phi_{f}^{i}$ or $\phi_{F}$ is greater than zero, since the value of this inherited portfolio weight determines the transaction costs to be paid at time 1. These preferences have been extensively used in empirical work by Grossman and Shiller (1981), Hansen and Singleton (1982), and many others.

\subsection{Optimization problem}

Facing a given return generating process, the investor can make unconditional (U) or conditional (C) portfolio choices. When making unconditional choices, the investor uses the steady state distribution and ignores any predictability of returns. In other words, the investor assumes returns are i.i.d. when making unconditional choices, which means the investor's unconditional choices would be optimal if returns were in fact i.i.d. with the unconditional distribution. In contrast, the investor exploits return predictability when making conditional choices. Thus, we can evaluate the impact of return predictability on portfolio choice when an investor faces proportional or fixed transaction costs.

We now present the Bellman equations associated with these two problems. For the conditional problem $(\mathrm{C})$, the fraction of portfolio value allocated to the risky assets at time $t, \alpha_{\mathbf{t}}$, is denoted by $\alpha\left(\mathbf{D}_{\mathbf{t}}, \hat{\alpha}_{\mathbf{t}}, \mathbf{t}\right)$, which is time dependent since the time horizon $T$ is finite. In contrast, if the investor ignores predictability $(\mathrm{U})$, the optimal allocation to the risky asset no longer depends on the $D_{t}$ state and so $\alpha\left(\hat{\alpha}_{t}, t\right)$ can denote $\alpha_{t}$.

\subsubsection{Conditional (C) Portfolio Choices}

Given our parametric assumptions, the Bellman equation faced by the investor is given by

$$
\begin{aligned}
\frac{a\left(D_{t}, \hat{\alpha}_{t}, t\right) W_{t}^{1-\gamma}}{1-\gamma}= & \max _{\kappa_{t}, \alpha_{t}}\left\{\frac{\kappa_{t}^{1-\gamma} W_{t}^{1-\gamma}}{1-\gamma}\right. \\
& \left.+\frac{\left(1-\kappa_{t}\right)^{1-\gamma} W_{t}^{1-\gamma}}{1-\gamma} E\left[a\left(D_{t+1}, \hat{\alpha}_{t+1}, t+1\right) R_{W, t+1}^{1-\gamma} \mid D_{t}, \hat{\alpha}_{t}\right]\right\},
\end{aligned}
$$

for $t=1, \ldots, T-1$

where $E[. \mid D, \hat{\alpha}]$ denotes the expectation taken using the conditional distribution given $D$. This form of the value function derives from the CRRA utility specification in eqs. (5), and from the linearity in $W$ of the budget constraint, eq.(2). 


\subsubsection{Unconditional (U) Portfolio Choices}

The Bellman equation faced by the investor is given by

$$
\begin{aligned}
\frac{a\left(\hat{\alpha}_{t}, t\right) W_{t}^{1-\gamma}}{1-\gamma}= & \max _{\kappa_{t}, \alpha_{t}}\left\{\frac{\kappa_{t}^{1-\gamma} W_{t}^{1-\gamma}}{1-\gamma}\right. \\
& \left.+\frac{\left(1-\kappa_{t}\right)^{1-\gamma} W_{t}^{1-\gamma}}{1-\gamma} E^{U}\left[a\left(\hat{\alpha}_{t+1}, t+1\right) R_{W, t+1}^{1-\gamma} \mid \hat{\alpha}_{t}\right]\right\},
\end{aligned}
$$

for $t=1, \ldots, T-1$.

where $E^{U}\left[. \mid \hat{\alpha}_{t}\right]$ denotes expectations taken assuming returns are i.i.d. with the same unconditional distribution as in the conditional case. This expectation does not depends on $D_{t}$, and neither does $\alpha_{t}$. Note that the Bellman eqs. (6) and (7) are solved by backward iteration, starting with $t=T-1$ and either $a(D, \hat{\alpha}, T)=1$ or $a(\hat{\alpha}, T)=1$.

\subsection{Utility comparisons}

Each of the investor problems described above imply a policy function that, in turn, yields a particular level of expected lifetime utility. Specifically, the policy functions $\left\{\alpha\left(\mathbf{D}_{\mathbf{t}}, \hat{\alpha}_{\mathbf{t}}, \mathbf{t}\right)\right\}_{\mathbf{t}=\mathbf{1}}^{\mathbf{T}-\mathbf{1}}$ and

$\left\{\kappa\left(D_{t}, \hat{\alpha}_{\mathbf{t}}, \mathbf{t}\right)\right\}_{\mathbf{t}=\mathbf{1}}^{\mathbf{T}-\mathbf{1}}$ can be substituted into the actual law of motion for investor's wealth eq. (1) to obtain the consumption sequence $\left\{c_{t}=\kappa\left(D_{t}, \hat{\alpha}_{\mathbf{t}}, \mathbf{t}\right) \mathbf{W}_{\mathbf{t}}\right\}_{\mathbf{t}=\mathbf{1}}^{\mathbf{T}}$. This consumption sequence is then substituted into eq. (5) to obtain the investor's expected lifetime utility.

In our utility comparisons we are interested in the fraction of wealth that an investor who is using a given policy, would be prepared to give up to be allowed to use an alternate policy. Four utility comparisons are performed. First, the utility cost of transaction costs are assessed. Second, the utility cost of holding one risky asset rather than another risky asset is assessed for all possible pairwise combinations of the risky asset portfolios. Third, the utility cost of holding one or other of the two risky assets rather than both is assessed. All three of these comparisons are performed with and without predictability. Finally, the utility cost of ignoring predictability is assessed for cases in which the investor has access to one or both risky assets. That is, in a world with predictability, we are interested in the fraction of wealth that an investor who is using the i.i.d. policy, would be prepared to give up to be allowed to use the optimal policy with predictability.

As mentioned above, the expected lifetime utility depends on the initial value of the inherited portfolio allocation, $\hat{\alpha}_{1}$, and the initial value of the vector characterizing the state of the economy, $D_{1}$. For simplicity, in the utility comparisons assessing the cost of ignoring predictability, we assume that, for a given $D_{1}, \hat{\alpha}_{1}$ equals the optimal $\alpha_{1}$ for the $\mathrm{U}$ case with zero transaction costs. In all 
other utility comparisons, $\hat{\alpha}_{1}$ for any investor problem is always set equal to the optimal $\alpha_{1}$ for analogous problem with zero transaction costs.

\subsection{Solution technique}

The dynamic programming problems are solved by backward recursion. With two risky assets, the state variables $\hat{\alpha}_{1}$ and $\hat{\alpha}_{2}$ are discretized and the value function is linearly interpolated between $\left(\hat{\alpha}_{1}, \hat{\alpha}_{2}\right)$ points. This technique yields an approximate solution that converges to the actual solution as the $\left(\hat{\alpha}_{1}, \hat{\alpha}_{2}\right)$ grid becomes finer. In all the optimizations, the holdings of both the risky and the riskless assets are constrained to be non-negative. In the two-risky asset case, this restricts the action space with respect to allocation choice to the triangular region characterized by $\alpha_{1}+\alpha_{2} \leq 1$, $\alpha_{1} \geq 0 \alpha_{2} \geq 0$. When actions are restricted to this set, implied inherited allocations for any return realization on the assets are again in the same region. We use the same discretization for state and action spaces and restrict holdings on each asset and the inherited allocations to lie on the discrete grid $\{0.00,0.01, \ldots, 0.99,1.00\}$. This gives a joint grid of 5151 points for the two assets. The value function is interpolated linearly for the implied inherited allocations by fitting a plane through the adjacent (as determined by the natural triangular tessellation of the space) 3 grid points. This solution technique specializes to the case with one risky asset in the natural way.

\section{Return Calibration}

We use the one-month Treasury-bill rate as a proxy for the risk-free rate $R^{f}$, and the 12 -month dividend yield on the value-weighted NYSE index as a proxy for the predictive variable $D$. We use three risky assets in various combinations as the set of available risky assets . The first is the monthly rate of return on the value-weighted NYSE index. The other two are low and high book-to-market portfolios. These two portfolios are formed from the six value-weighted portfolios SL, SM, SH, BL, BM, and BH from Fama and French (1993) and Davis, Fama and French (1999). The notation S (B) indicates that the firms in the portfolio are smaller (larger) than 50 percent of NYSE stocks. The notation L indicates that the firms in the portfolio have book-to-market (B-M) ratios that place them in the bottom three deciles for all stocks; analogously, M indicates the middle four deciles and $\mathrm{H}$ indicates the top three deciles. The high B-M portfolio is an equally-weighted portfolio of $\mathrm{SH}$ and $\mathrm{BH}$ while the low B-M portfolio is an equally-weighted portfolio of SL and BL. The three stock returns and interest rate series are deflated using monthly CPI inflation. We calibrate real returns since they are more likely to be stationary, investors generally care more about 
real returns, and there is no money in our model. The interest rate and dividend yield series are from CRSP, the CPI series is from CITIBASE. and the three stock portfolios are constructed from data on Ken French's website. The data period used is from 1927:7 to 1996:11. The continuously compounded riskfree rate is estimated to be the mean of the continuously compounded one-month Treasury-bill rate over this period, which gives a value for $R^{f}$ of 0.042 percent.

Assume that $\mathbf{R}$ is an $N \times 1$ return vector and let $\mathbf{r} \equiv \ln (\mathbf{1}+\mathbf{R})$ and $d \equiv \ln (1+D)$. We assume that $\left[\mathbf{r}^{\prime} d\right]^{\prime}$ follows the vector autoregressive model (VAR):

$$
\begin{gathered}
\mathbf{r}_{\mathbf{t}+\mathbf{1}}=\mathbf{a}_{\mathbf{r}}+\mathbf{b}_{\mathbf{r}} \mathbf{d}_{\mathbf{t}}+\mathbf{e}_{\mathbf{t}+\mathbf{1}}, \\
d_{t+1}=a_{d}+b_{d} d_{t}+v_{t+1},
\end{gathered}
$$

where $\mathbf{a}_{\mathbf{r}}, N \mathrm{x} 1$, and $a_{d}$ are intercepts, $\mathbf{b}_{\mathbf{r}}, N \mathrm{x} 1$, and $b_{d}$ are coefficients and $\left[\mathbf{e}^{\prime} v\right]^{\prime}$ is an i.i.d. vector of mean-zero, multivariate normal disturbances, with constant covariance matrix $\boldsymbol{\Sigma}_{\mathbf{e v}, \mathbf{e v}}$; the covariance matrix of $v$ is $\boldsymbol{\Sigma}_{\mathbf{v}, \mathbf{v}}$ and the variance of $e$ is $\sigma_{e}^{2}$. Similarly, the unconditional covariance matrix for $\left[\mathbf{r}^{\prime} d\right]^{\prime}$ is $\boldsymbol{\Sigma}_{\mathbf{r d}, \mathbf{r d}}$; the unconditional variance matrices for $\mathbf{r}$ and $d$ are $\boldsymbol{\Sigma}_{\mathbf{r}, \mathbf{r}}$ and $\sigma_{d}^{2}$ respectively. Without loss of generality, we normalize the mean of $d, \mu_{d}$, to be zero and its variance, $\sigma_{d}^{2}$, to be 1. Specification (8)-(9) assumes that $d_{t}$ is the only state variable needed to forecast $\mathbf{r}_{\mathbf{t}+\mathbf{1}}$ which is in line with other papers on optimal portfolio selection (e.g., Barberis (1999) and Campbell and Viceira (1996)).

The data VAR is estimated using ordinary least squares (OLS) and discretized using a variation of Tauchen and Hussey's (1991) Gaussian quadrature method; the variation is designed to ensure that $d$ is the only state variable (see Balduzzi and Lynch (1999) for details). However, following Lynch (2000), this study implements the discretization in a manner that produces exact matches for important moments for portfolio choice. In particular, the procedure matches both the conditional mean vector and the covariance matrix for log returns at all grid points of the predictive variables, as well as the unconditional volatilities of the predictive variables and the correlations of log returns with the predictive variables. We choose 19 quadrature points for the dividend yield and 3 points for the stock-return innovations since Balduzzi and Lynch (1999) find that the resulting approximation is able to capture important dimensions of the return predictability in the data.

Table 1 presents VAR parameter values for both the data and the various quadrature approximations used. Panel A reports the slope coefficients $\mathbf{b}_{\mathbf{r}}$ and $b_{d}$ as well as unconditional means for $\mathbf{r}$ and $d$. Panel B reports the unconditional covariance matrix for $\left[\mathbf{r}^{\prime} d\right]$ and and the cross-correlations. Panel C reports the unconditional covariance matrix for $\left[\mathbf{e}^{\prime} v\right]$ and the cross-correlations. Throughout the table, the quadrature values almost always replicate the data values, which suggests that 
the discretization is capturing the important features of the data. Further evidence on this point can be found in Balduzzi and Lynch (1999).

\section{Risky-asset Pairs and Parameter choices}

We examine decision-making in the presence of transaction costs when the investor has access to either of two pairs of assets: 1) the market and the high B-M assset; and 2) the market and the low B-M asset. We examine portfolio rebalancing rules for the unconditional and conditional problems. For each pair, the effects of losing access to one of those risky assets and the effects of predictability are also assessed.

The investor's risk aversion parameter, $\gamma$, is set to eight throughout. This $\gamma$ choice is motivated by the Mehra and Prescott (1985) argument that the existing evidence from macro and micro studies constitutes an a priori justification for restricting the value of $\gamma$ to be less than ten. The horizon $T$ of the young investor is 240 periods or 20 years, since the return processes are calibrated to monthly returns. A 20-year horizon is a realistic investment horizon for an investor who retires at time 1 . The time preference parameter, $\delta$ is set equal to the inverse of the riskfree return.

The proportional transaction cost parameters, the $\phi_{p}$ s, are set equal 0.25 percent for the market, 0.5 percent for the high B-M asset and 0.375 percent for the Low B-M asset. The value for the market $\phi_{p}$ implies a round-trip transaction cost of 0.5 percent. Both Fidelity's Spartan 500 Index Fund and the Schwab S\&P 500 Index Fund attempt to track the S\&P 500 index, and as of the 22nd of August 2002, the former charges a redemption fee of 0.5 percent on fund shares sold within three months of purchase while the latter charges a redemption fee of 0.75 percent on fund shares sold within six months of purchase. ${ }^{5}$ Turning to the book-to-market assets, Fidelity's structured midcap growth and value funds both charge a redemption fee of 0.75 percent on fund shares sold within one month of purchase. However, funds that invest in small firms typically charge higher redemption fees than this. For example, Fidelity's small cap stock fund charges a 2 percent redemption fee on fund shares sold within three month of purchase. Our high and Low B-M assets have 50\% invested in small firms, but the high B-M asset is likely to be holding a much larger number of small firms than the Low B-M asset (see Tables II and IV in Fama and French, 1992). For this reason, the $\phi_{p}$ parameter is set slightly higher for the high B-M asset than the Low B-M asset. While our proportional cost structure does not capture the specific features of these redemption fees, our

\footnotetext{
${ }^{5}$ Similarly, Lynch and Balduzzi (2000) report that as of the 5th of May 1999, both Fidelity's Spartan Total Market Index Fund and the Schwab Total Market Index Fund charge a redemption fee of 0.5 percent on fund shares sold within three and six months respectively of purchase.
} 
chosen values for the $\phi_{p}$ s can be viewed as indicative of the proportional rebalancing costs faced by an individual using index and mutual funds to hold the equity portfolios we consider.

The fixed cost parameters, $\phi_{f}$ for rebalancing the market, $\phi_{f}$ for rebalancing the high B-M, $\phi_{f}$ for rebalancing the Low B-M, and $\phi_{F}$ for any rebalancing at all, are all taken to be 0.01 percent. This value translates into paying a fee of $\$ 10$ whenever a $\$ 100,000$ portfolio is reshuffled in such a way as to trigger the particular fixed cost. The cases considered never simultaneously have non-zero $\phi_{F}$ and $\phi_{f}$ s. Viewed as the opportunity cost for an individual to process information and instruct a broker to change portfolio composition, this value for $\phi_{F}$ and the $\phi_{f}$ s seems small.

\section{Portfolio choice results}

This section reports portfolio rebalancing rules when the investor faces transactions costs and has access to two risky assets. We start with i.i.d. returns and then move to the case with return predictability.

We present rebalancing rules for return generating processes calibrated to the data. We also present rebalancing rules for return generating processes that perturb the data process in a way that allows us to better understand how the return generating process affects the rebalancing rules. In particular, it seems that return correlation is most important for i.i.d. returns and that conditional return correlation is most important for predictable returns. In the i.i.d. return cases, we take the data-calibrated discretization and rotate the returns using the Cholesky decomposition of the covariance matrix, to obtain returns with the same means and variances but the desired correlation. Turning to the allocations with return predictability, we consider three cases, one that matches the conditional correlation to the data, another that sets the conditional correlation to zero in all states, and a third that allows the conditional correlation to vary across states. For the case with zero conditional correlation, we use the Tauchen and Hussey procedure as in the data case but calibrate a conditional covariance matrix with zero return correlation instead of the one implied by the data. For the case with state-dependent correlation, we start with the data-calibrated discretization and then perform a return rotation state-by-state to obtain the desired conditional return correlation for each state.

\subsection{Unconditional allocations}

Figures 1 through 5 plot rebalancing rules for a variety of transaction cost structures when the investor has access to the high asset and the market, and returns are i.i.d.. Each figure reports 
rebalancing rules for the investor's last period in graphs a through $\mathrm{d}$ and for the investor's first period in graphs e through h. Rebalancing rules for four return generating processes are plotted. Graphs a and e plot the case of zero correlation between the two risky returns while graphs b and $\mathrm{f}$ plot the case in which the risky asset correlation is matched to that in the data. Since the data correlation induces a no-trade region that touches the boundary, graphs $\mathrm{d}$ and $\mathrm{h}$ plot the case of correlation that is $30 \%$ of that in the data. Lastly, graphs c and g plot a negative correlation case in which the correlation is negative $30 \%$ of that in the data. Unconditional means and variances for the two risky assets are held constant across the four return generating processes.

Figure 1 plots rebalancing rules for the case with proportional costs but no fixed costs. The rebalancing rule is a no-trade region whose shape depends on the correlation between the two risky asset returns. When the correlation is zero in graphs 1.a and 1.e, the no-trade region is a rectangle, consistent with Liu (2002) who finds a similar result for exponential utility. When the correlation is non-zero, the no-trade rectangle becomes a no-trade parallelogram, which suggests that a similar finding by Leland is robust to using a utility-based objective function for the investor. With the positive correlation in graphs 1.d and 1.h, the parallelogram distorts the associated rectangle in such a way as to take advantage of the associated substitutability across the two assets that the positive correlation induces. The converse is true for negative correlation, as graphs 1c and 1.g demonstrate.

Once the inherited allocation pair lies outside the no-trade region, the investor rebalances to one of the boundaries. When the no-trade region is a rectangle, the investor rebalances to the closest boundary and so only rebalances both risky assets when a rectangle corner is the closest boundary point. A corner is the closest boundary point whenever the inherited allocation for each asset is not an allocation for that asset anywhere in the no-trade region. When the no-trade region is a parallelogram, it remains true that that the investor only rebalances both risky assets whenever the inherited allocation for each asset is not an allocation for that asset anywhere in the no-trade region. Each pair of opposite sides of the parallelogram are associated with rebalancing of the same asset. For a given pair of opposite sides, an inherited allocation pair with an allocation to the other asset equal to a point on the closer of the two sides moves to that boundary point.

There is intuition for why the optimal rebalancing rule utilizes a no-trade region. For each risky asset, the partial derivative of the proportional cost function with respect to a change in the asset's portfolio weight is equal to the asset's cost parameter for any positive weight change and is equal to the negative of this parameter for any negative weight change. Thus, this derivative 
exhibits a discontinuity when evaluated at a weight change of zero. At the same time, the utility loss associated with the deviation of an asset's portfolio weight from its optimal no-transactioncost weight (expressed as a negative value) is a concave function of the deviation, whose derivative equals zero at a zero deviation. Consequently, if the investor faces a proportional cost, she only rebalances an asset if its weight is sufficiently far away from its no-transaction-cost optimum that the benefit from rebalancing even a little exceeds the associated transaction cost.

Comparing the first four graphs to the last four, it is clear that the no-trade region shrinks as the investor gets younger. This result is consistent with the findings of Lynch and Balduzzi (2000) for the single risky asset case and the intuition is the same. Late in life, the benefits of rebalancing are capped by the terminal date, whereas early in life, the benefits are enjoyed for several months after the rebalancing occurs. Greater benefits from rebalancing early in life translate into smaller no-trade regions.

Figure 2 plots rebalancing rules for the case with proportional costs and a fixed cost associated with each asset that is triggered by rebalancing that asset. Again, the no-trade region is a rectangle when the correlation is zero, and a parallelogram otherwise. Because of the fixed cost, the rebalancing-return curve now lies inside the no-trade boundary. Moreover, once the fixed cost of rebalancing is triggered, it is a sunk cost and so the rebalance-return allocation for that asset in the last period (graphs a through d) is equal to that in the case with only proportional costs. In particular, in the last period, if the investor rebalances both assets despite the fixed costs, the return point is the same as in the proportional cost case. Similarly, in the last period, if the investor rebalances the same asset as in the proportional cost case, the return point is also the same as in the proportional cost case. However, there are some inherited allocations for which the fixed cost cause the investor to rebalance fewer risky assets than in the proportional cost case.

Figure 3 plots rebalancing rules for the case with proportional costs and a fixed cost that is triggered by rebalancing only one risky asset or both. The no-trade region is no longer a rectangle or parallelogram. Instead, the corners are now rounded due to the fixed cost being triggered by any rebalancing. However, the distortions in the no-trade region due to correlation are again consistent with the substitution and complement effects expected to be associated with positive and negative correlation respectively. In the last period, once the fixed cost is triggered, the investor rebalances exactly as she would if there were just proportional costs.

Figures 4 and 5 plot rebalancing rules for two cases that set proportional costs to zero. In Figure 4, the fixed costs are the same as in Figure 2, with a fixed cost for each asset that is trig- 
gered by rebalancing that asset. When the correlation is zero, the no-trade region is a rectangle, both late and early in life. When the correlation is non-zero, the no-trade regions are not exactly parallelograms with the corners slightly rounded. The distortions from a rectangle are consistent with the substitution and complement effects expected to be associated with positive and negative correlation respectively. Sometimes the investor only rebalances one asset, which is consistent with a separate fixed cost associated with rebalancing each asset. But when the investor rebalances only one asset, the rebalance-return allocation is solely determined by the other asset's inherited allocation. The rebalance-return allocations when both assets are rebalanced are the same irrespective of the inherited allocations. These results are to be expected given the absence of proportional costs. When both assets are rebalanced, the return allocations are exactly those for the case with no transaction costs. While this result is expected for the last period of life, it is surprising that the result is also obtained for all other periods as well. In figure 5, the fixed costs are the same as figure 3, with a single fixed cost that is triggered by rebalancing either or both assets. With a single fixed cost, the no-trade region is oval-shaped and if the investor rebalances, the return allocations are the same irrespective of the inherited allocations. And irrespective of the period of life, the rebalance-return allocations are the same as in the optimal allocations in the case with no transaction costs.

Focussing on the case in which the two asset returns have the same correlation as in the data (always graphs b and $\mathrm{f}$ in Figures 1-5), the optimal allocations with no transaction costs are about 26 percent in the high B-M asset and nothing in the market. In the last period, transaction costs cause the investor to implement sizeable no-trade regions and rebalance-return curves that imply positive holdings of the market. For example, with proportional costs only and a large inherited allocation to the market, Figure 2.b indicates that the investor may prefer to keep as much as 35 percent allocated to the market, depending on the inherited allocation to the high B-M. However, these results may be a little misleading since early in life, the investor rebalances to a zero holding of the market in all five cases considered. Consequently, the investor will start her life by selling any inherited high B-M asset. Moreover, even in the last period, an inherited allocation to the market of zero always results in a chosen allocation in the market of zero. So after any initial selloff, the investor keeps a zero allocation in the market over her entire life. 


\subsection{Conditional allocations}

Figures 6 and 7 plot rebalancing rules when the investor has access to the Low B-M asset and the market, and faces proportional transaction costs only. Returns are either predictable or i.i.d.. Each figure reports rebalancing rules for the investor's last period in graphs a through d and for the investor's first period in graphs e through h. With predictable returns, rebalancing rules for three dividend-yield states are plotted: the lowest dividend-yield state (state 1) is plotted in graphs a and e; the middle dividend-yield state (state 10) is plotted in graphs b and f; and the highest dividend-yield state (state 19) is plotted in graphs c and g. The i.i.d. case is plotted in graphs d and h. For each figure, the unconditional distribution is the same for the predictable and the i.i.d. cases.

Figure 6 plots rebalancing rules for the case in which the risky asset correlation is matched to that in the data. In the last period of life (graphs a through d), the no-trade regions, in all but the lowest dividend-yield state, have two boundaries that are negative-sloped lines running from the vertical axis to the horizontal axis. The positive correlation between the two returns is causing a highly distorted no-trade region relative to a rectangle. The substitutability of the two assets causes the investor to accept a lower inherited allocation of one asset if accompanied by a compensatingly higher inherited allocation of the other asset.

One interesting question is whether the shape of the no-trade region, state by state with predictability, is the same as in the i.i.d. return case. But just by looking at the last period rules, it is difficult to tell whether the no-trade regions are parallelograms, state by state, in the return predictability case. The reason is that the no-trade regions are so large that they intersect the short-selling constraints. In fact, from Figure 6.d, it is hard to see from the last period rules that the no-trade region is a parallelogram even in the i.i.d. case

But in the first period of life, the no-trade regions are much smaller and the no-trade region for the highest dividend state in Figure 6.g is clearly a parallelogram. Moreover, in unreported results, moving from the highest to the middle dividend-yield states, the no-trade region shifts downward but always looks like a parallelogram that is being truncated by the horizontal axis. Thus, our results suggest that when returns are predictable, proportional costs induce no-trade regions state by state that are parallelograms. Consequently, the no-trade regions state by state with predictability have the same shape as the no-trade regions with i.i.d. returns, which generalizes the result in Balduzzi and Lynch (1999) for the case with a single risky asset.

With i.i.d. returns, the nature of the no-trade parallelogram's distortion relative to the asso- 
ciated rectangle depends on the risky-asset return correlation. In particular, the knife-edge case is zero correlation which leads to no distortion and a no-trade rectangle. It is worth asking what determines the distortion in the case of predictable returns. Intuition suggests that conditional correlation is the critical variable. To examine this conjecture, Figure 7 plots rebalancing rules for the case in which the risky asset conditional correlation is set to zero; the i.i.d. case with the same unconditional correlation is plotted in graphs $\mathrm{d}$ and $\mathrm{h}$. To keep the conditional contemporaneous covariance matrix for returns and dividend yield positive definite, the conditional return correlations with the dividend yield are scaled to be 75 percent of those in the data. The no-trade regions both in the last and first periods are always either rectangles or truncated rectangles. This finding is strongly suggestive that conditional return correlation determines the shape of the no-trade region when returns are predictable and transaction costs are proportional.

To further explore this issue, the homoscedastic VAR assumption is relaxed so that conditional return correlations can vary across dividend states. In particular, the conditional correlations are calibrated to be negative for the low dividend-yield states, positive for the high dividend-yield states, and zero for the middle state. Figure 8 plots the resulting rebalancing rules. When the conditional return correlation for a state is zero as in graphs c and $\mathrm{f}$, the no-trade region is a rectangle both early and late in life. When the conditional return correlation for a state is positive as in graphs $\mathrm{b}$ and $\mathrm{e}$, the no-trade region is a parallelogram which is a rectangle distortion that takes advantage of the asset substitutability associated with positive return correlation. Finally, when the conditional return correlation for a state is negative as in graphs a and d, the no-trade region is a parallelogram which is a rectangle distortion that takes advantage of the asset complementarity associated with negative return correlation.

The numerical results in Figures 7 and 8 strongly suggest that with return predictability, conditional correlation determines the nature of the distortion to the no-trade parallelogram. In particular, it is the conditional return correlation in a given state that determines the distortion to the parallelogram in that state. Moreover, this result is robust to the presence of return heteroscedasticity and holds early as well as late in late. For example, even when the conditional correlations are non-zero in all other states, the investor still uses a no-trade rectangle in the all periods in a state with zero conditional return correlation.

We documented above for the i.i.d. return case how the no-trade region shrinks as the investor gets younger. It is interesting to examine whether this shrinkage still occurs when returns are predictable. Examining Figures 7, it is clear that the no-trade regions shrink much less when 
returns are predictable than when returns are i.i.d., holding the unconditional distribution fixed. For the i.i.d. case, the dimensions of the no-trade rectangle shrink from $21 \%$ by $23 \%$ in the last period (Figure 7.d) to $5 \%$ by $5 \%$ in the first (Figure 7.h). In contrast, when returns are predictable, the average dimensions across states whose no-trade rectangle is not truncated by the short-selling constraints goes from $20.6 \%$ by $22.4 \%$ in the last period to $8.6 \%$ by $9.1 \%$ in the first. Thus, the difference in the size of the no-trade region between early and late in life is less pronounced when returns are conditional, consistent with intuition that the benefits from rebalancing today are more short-lived when returns are predictable than in the i.i.d. case. In particular, as the dividend-yield state moves around through time, the benefits of today's rebalancing become smaller and smaller, since the optimal allocation is shifting with the dividend-yield state. It is also worth noting that the similar sizes of the no-trade regions across the i.i.d. and predictability cases in the last period is to be expected, given that the conditional return variances are very similar to the return variances in the i.i.d. case (see Table 1).

\section{$6 \quad$ Utility calculations}

This section discusses the utility comparison results reported in Tables 2 through 5 .

\subsection{The utility cost of transactions costs}

Table 2 reports the utility costs associated with various transaction costs structures relative to the no transaction cost case. Reported numbers are the wealth percentages that the investor would be prepared to give up to not pay the applicable transaction costs. For the predictability cases, the number reported is a mean wealth percentage whose standard deviation is in parentheses below. ${ }^{6}$ The first row reports results for proportional costs only, while the second reports numbers for the case with proportional costs and a single fixed cost. The final row reports wealth percentages for the case with a proportional and a fixed cost associated with each asset. Consequently, the cost structures become increasingly more onerous moving from the top to the bottom row. There are five columns of numbers each corresponding to a risky-asset set that the investor could have access to. In each of the first three columns, one of the risky assets is available individually, while each of the last two columns correspond to a risky-asset pair.

The utility costs increase going down any column, which is expected given the increasing severity

\footnotetext{
${ }^{6}$ The means and standard deviations for the wealth percentage are always calculated using the unconditional distribution for the dividend yield.
} 
of the transaction costs going from the top to the bottom row. For a given risky-asset set and cost structure, the cost of the transaction costs is always much higher when returns are predictable than when returns are i.i.d.. This result is consistent with the investor rebalancing much more when returns are predictable to take advantage of the timing possibilities that predictability offers.

Comparing the first two rows, the utility costs are much higher once a single fixed cost is added to the proportional costs. But the similarity of the wealth percentages across the last two rows indicates that the imposition of an additional fixed cost for trading both assets does not translate into much of a utility loss. For three of the four cases, the utility difference is indistinguishable from zero and this is because of rebalancing rules that induce the investor to never (or almost never) hold one of the risky assets. When the investor has access to the market and the high B-M asset, the investor never holds the market when returns are i.i.d., and almost never holds the market when returns are predictable. When the investor has access to the market and the low B-M asset, the investor never holds the low B-M asset when returns are i.i.d.. If the investor never holds one of the assets, she never needs to rebalance the two assets at the same time and hence the second fixed cost is never incurred. Turning to the fourth case, which is the market and the low B-M asset with predictability, unreported results show that the investor holds both assets in 6 of the 19 states. We know from Figures 2 and 3 that the presence of a second fixed cost alters the investor's rebalancing rule. So in this fourth case, this adjustment to the rebalancing rule must allow the investor to largely neutralize the effect of the second fixed cost, at least for the fixed cost imposed here. This result is reminiscent of the Constantinides (1986) result for proportional costs, which says that the imposition of a proportional cost has only a second-order effect on utility because the investor rebalances less frequently when faced with such a cost.

\subsection{Utility associated with accessing one risky asset rather than another}

Table 3 performs a utility comparison for the three possible risky asset pairs by reporting the wealth percentage that the investor with access to one of the risky assets would be willing to give up to obtain access to the other. The first asset risky asset in each column heading is the one the investor is giving up. When the investor is given access to a risky asset, the initial value of the inherited portfolio allocation is set equal to the optimal beginning-of-horizon portfolio allocation for the same asset without transactions costs. Based on the results in Table 3, when the investor can only hold one of the risky assets, the investor receives the highest utility if that asset is the high B-M asset and the lowest utility if that asset is the low B-M asset. This ranking holds irrespective of whether 
returns are i.i.d. or predictable, and irrespective of the transaction cost structure.

When comparing either the market or the low B-M asset to the high B-M asset, the wealth percentage that the investor would be prepared to give up to get access to the high B-M asset is increasing in the severity of the transaction cost structure. In contrast, the wealth percentage that an investor holding the low B-M asset would give up to get access to the market instead is decreasing in the transaction cost severity. Both these results obtain with or without predictability. In the i.i.d. case, these seemingly contradictory findings can be reconciled by examining the return volatilities reported in Table 1. The high B-M asset has the highest volatility while the market has the lowest. Transaction costs have a greater impact on utility when return volatility is higher, since the implication is more rebalancing (see Constantinides, 1986). Thus, since the high B-M asset has the highest variance, its advantage over the other two assets is eroded by transaction costs. When going from no transaction costs to only proportional costs, the fact that the high B-M asset also has the highest proportional cost parameter is also playing a role. In contrast, the market has a lower volatility than the low B-M asset so its advantage is magnified by the presence of transaction costs. Again, the fact that the market has a lower proportional cost than the low B-M asset is also playing a role in this magnification.

Turning to the case with return predictability, the same arguments as in the i.i.d. case continue to hold though intuition suggests that it is the conditional volatility that matters. Even so, the conditional volatility ranking for these three assets is exactly the same as the ranking based on unconditional volatility. However, there is an additional force at work with predictable returns. Return predictability induces rebalancing as the investor takes advantage of the timing opportunities that the predictability generates. Consequently, transaction costs can be expected to have a greater adverse affect when the available risky asset is more predictable. Table 1 shows that the predictive regression slopes and R-squareds give the same ranking, with the high B-M asset having the most predictable return and the market the least. This greater predictability is another reason why the high B-M asset's advantage is eroded by more severe transaction costs. And the lesser predictability of the market relative to the low-B-M asset is another reason why the market's advantage relative to the low-B-M asset is exacerbated by higher transaction costs.

The predictability ranking of the three risky assets also helps explain the pattern of results going from i.i.d. to predictable returns. In particular, the high B-M asset's advantage relative to the other two assets increases when returns are predictable while the market's advantage relative to the low B-M asset decreases. Since the high B-M asset's return is the most predictable, intuition 
suggests that its advantage would become even greater once return predictability was allowed. In contrast, since the market's return is the least predictable, one would expect its advantage relative to the low B-M asset to be smaller once return predictability was allowed.

\subsection{The utility cost of accessing only one of the two risky assets}

Table 4 reports the percentage wealth that an investor with access to a single risky asset would be willing to sacrifice to gain access to a second risky asset. Again, when returns are predictable, the table reports the mean wealth percentage, with the standard deviation in parentheses below. Generalizing the approach in Table 3 above, the initial value of the inherited portfolio allocation for a given set of assets is set equal to the optimal beginning-of-horizon allocation for the set without transactions costs.

The wealth percentage that the investor is willing to give up to add the market to the high B-M asset is exactly zero when returns are i.i.d. and almost zero when returns are predictable. The implication is that, with access to both assets, the investor never holds the market in the i.i.d. return case and almost never holds the market when returns are predictable. This conclusion is confirmed by comparing the cost of having access to the market instead of the high B-M asset (see Table 3) to the cost of having access to the market instead of both. In the i.i.d. case, these two costs are the same, while in the predictability case, the latter cost is only slightly higher than the former. Turning to the market and low B-M pair, the cost of having access to the market rather than both is exactly zero in the i.i.d. case which again indicates that the investor never holds the low B-M asset when she has access to both. As above, the cost of having access to the low B-M asset and not the market (see Table 3 ) is exactly the same as the cost of having access to the low B-M asset instead of both. However, when returns are predictable, the cost of having access to the market rather than both is above zero, which suggests that the investor is holding both assets in non-trivial amounts.

Utility cost patterns as a function of transaction cost severity and whether returns are predictable or not can be explained using analogous arguments to those presented to explain such patterns in Table 3. Since having access to the market-high B-M pair induces essentially the same holdings as having access to the high B-M asset, the patterns in the advantage of the high B-M asset over the market in Table 3 must be the same as the patterns in the advantage of having both rather than just the market reported in Table 4. The explanations must be the same too. Turning to the market and low B-M pair, the same situation exists in the i.i.d. case. The market-low B-M 
pair induces essentially the same holdings as having access to the market alone. However, when returns are predictable, the investor holds non-trial amounts of the market and the low B-M asset. Going from the no transaction cost case to the case with both a proportional and a single fixed cost, the wealth percentage given up to access both assets is decreasing in the transaction cost severity when starting with the market but is increasing when starting with the low B-M portfolio. This change in direction depending on the starting asset is likely due to the optimal combination of the two assets having volatility and/or predictability that are lower than the low B-M asset but higher than the market. Such a switch would explain the change since transaction costs have a larger effect on utility whenever return volatility or predictability is higher. Finally, the presence of a further fixed cost to trade the second risky-asset does not affect the investor with access to only one risky asset but it does adversely affect the investor when she has access to both. Consequently, comparing the third and fourth rows of Panel B, the utility cost of not using the second asset always weakly increases with the addition of a separate fixed cost to trade the second asset.

Return predictability reduces the utility cost associated with having access to the low B-M portfolio rather than the market (see Table 3) but increases the utility cost associated with having access to the low B-M portfolio instead of both assets. This result is consistent with intuition. In particular, the optimal portfolio of the low B-M asset and the market is likely to have a more predictable return than the low B-M asset alone, even though this low B-M asset has a return that is more predictable than the market's return.

\subsection{The utility cost of ignoring predictability}

Table 5 reports the mean and standard deviation (in parentheses) of the fraction of wealth that an investor would be willing to sacrifice to gain access to predictive information concerning risky asset returns. For the predictability cases, the initial values of the inherited portfolio allocations are set equal to the optimal allocations for the associated i.i.d. cases with zero transaction costs. The utility cost of ignoring risky asset return predictability is almost always slightly decreasing in transactions costs. For example, when the CRRA investor has access to the market portfolio, the utility cost is $4.24 \%$ when there are no transactions costs, $4.12 \%$ when there are only proportional transactions costs, and $4.05 \%$ when there are both fixed and proportional transactions costs. This result is consistent with intuition since transactions costs are frictions that prevent the investor from adjusting her portfolio to take advantage of return predictability. In the limit, when transactions costs are sufficiently high to prevent any trading, there is no utility loss associated with ignoring 
return predictability.

Examining the cost of ignoring predictability when the investor has access to only one asset, we see that the cost is highest when the investor has access to the high B-M asset only and lowest when the investor has access to the market. By giving the investor access to two risky assets, we can examine how the cost of ignoring predictability is affected by increased access to the asset market. When the investor has access to the market and the high B-M asset, the investor almost always holds only the high B-M asset, with or without predictability. Consequently, the cost of ignoring predictability with access to these two assets is the same as with access to just the high B-M asset. In contrast, when the investor has access to the market and the low B-M asset and returns are predictable, the investor chooses to hold both assets in many of the dividend-yield states. Table 5 shows that the utility cost of ignoring predictability when the investor has access to both these assets simultaneously lies somewhere between the costs of ignoring predictability when the investor has access to each asset separately.

\section{Conclusions}

Our paper contributes to the dynamic portfolio choice and transaction cost literatures by considering a multiperiod individual who faces transaction costs and who has access to multiple risky assets, all with predictable returns. We numerically solve the individual's multiperiod problem in the presence of transaction costs and predictability. In particular, we characterize the investor's optimal portfolio choice with proportional transaction costs, and with return predictability similar to that observed for the U.S. stock market. We also perform some comparative statics to better understand the nature of the no-trade region with more than one risky asset. Throughout our focus is on the case with two risky assets. We also perform some utility comparisons. 


\section{References}

Akian, M., Menaldi, J.L. and Sulem, A., 1996. On an Investment-Consumption model with transaction costs. SIAM Journal of Control and Optimization 34, 329-364.

Balduzzi, P., Lynch, A., 1999. Transaction costs and predictability: some utility cost calculations. Journal of Financial Economics 52, 47-78.

Barberis, N., 2000. Investing for the long run when returns are predictable. Journal of Finance $55,225-264$.

Bhardwaj, R., Brooks, L., 1992. The january anomaly: effects of low share price, transaction costs and bid-ask bias. Journal of Finance 47, 553-574.

Brennan, M., Schwartz, E., 1996. The use of treasury bill futures in strategic asset allocation programs. Unpublished working paper. UCLA, Los Angeles, CA.

Brennan, M., Schwartz, E., Lagnado, R., 1997. Strategic asset allocation. Journal of Economic Dynamics and Control 21, 1377-1403.

Campbell, J., 1987. Stock returns and the term structure. Journal of Financial Economics 18, 373-99.

Campbell, J. Y. , Chan, Y. L. and Viceira, L. M.,2002. A multivariate model of strategic asset allocation. forthcoming Journal of Financial Economics.

Campbell, J., Viceira, L., 1999. Consumption and portfolio decisions when expected returns are time varying. Quarterly Journal of Economics 114, 433-495.

Constantinides, G., 1979. Multiperiod consumption and investment behavior with convex transaction costs. Management Science 25, 1127-1137.

Constantinides, G., 1986. Capital market equilibrium with transaction costs. Journal of Political Economy 94, 842-62.

Davis, M., Norman, A., 1990. Portfolio selection with transaction costs. Mathematics of Operations Research 15, 676-713. 
Duffie, D., Sun, T., 1990. Transaction costs and portfolio choice in a discrete-continuous-time setting. Journal of Economic Dynamics and Control 14, 35-51.

Fama, E., French, K., 1989. Business conditions and expected returns on stocks and bonds. Journal of Financial Economics 25, 23-49.

Fama, E., French, K., 1992. The cross-section of expected stock returns. Journal of Finance 47, 427-465.

Fama, E., French, K., 1993. Common risk factors in the returns on stocks and bonds. Journal of Financial Economics 33, 3-56.

Fleming, J., Ostdiek, B., Whaley, R., 1995. Trading costs and the relative rates of price discovery in stock, futures, and option markets. Unpublished working paper. Duke University, Durham, NC.

Grossman, S., Shiller, R., 1981. The determinants of the variability of stock market prices. American Economic Review 71, 222-227.

Hansen, L., Heaton, J., Luttmer, E., 1995. Econometric evaluation of asset pricing models. Review of Financial Studies 8, 237-274.

Hansen, L., Singleton, K., 1982. Generalized instrumental variables estimation of nonlinear rational expectations models. Econometrica 50, 1269-1286.

He, H., Modest, D., 1995. Market frictions and consumption-based asset pricing. Journal of Political Economy 103, 94-117.

Heaton, J., Lucas, D., 1996. Evaluating the effects of incomplete markets on risk sharing and asset pricing. Journal of Political Economy 104, 443-487.

Hodrick, R., 1992. Dividend yields and expected stock returns: alternative procedures for inference and measurement. Review of Financial Studies 5, 357-386.

Kandel, S., Stambaugh, R., 1996. On the predictability of stock returns: an asset allocation perspective. Journal of Finance 51, 385-424.

Kim, T., Omberg, E., 1996. Dynamic nonmyopic portfolio behavior. Review of Financial Studies 9, 141-161. 
Koo, H., 1991. Portfolio choice with linear transaction costs: theory, numerical solutions and applications. Unpublished working paper. Princeton University, Princeton, NJ.

Leland, H., 2000. Optimal Portfolio Implementation with Transactions Costs and Capital Gains Taxes. Unpublished working paper. University of California, Berkeley.

Liu, H., 2002. Optimal Consumption and Investment with Transaction Costs and Multiple Risky Assets. forthcoming Journal of Finance.

Lesmond, D., Trzcinka, T., Ogden, J., 1999. A new measure of total transaction costs. Review of Financial Studies, Vol 12, no. 5, pp. 1113-1141.

Lynch, A. W., 2001. Portfolio Choice and Equity Characteristics: Characterizing the Hedging Demands Induced by Return Predictability. Journal of Financial Economics 62, 67-130.

Lynch A. W., Balduzzi, P., 2000. Predictability and Transaction Costs: The Impact on Rebalancing Rules and Behavior. Journal of Finance 55, 2285-2310.

Luttmer, E., 1996a. Asset pricing in economies with frictions. Econometrica 64, 1439-1467.

Luttmer, E., 1996b. Consumption and portfolio choice in the presence of fixed costs. Unpublished working paper. Northwestern University, Chicago, IL.

Mehra, R., Prescott, E., 1985. The equity premium: a puzzle. Journal of Monetary Economics 15, $145-161$.

Morton, A., Pliska, S., 1993. Optimal portfolio management with fixed transaction costs. Unpublished working paper. University of Illinois at Chicago, Chicago, IL.

Samuelson, P., 1969. Lifetime portfolio selection by dynamic stochastic programming. The Review of Economics and Statistics 51, 239-246.

Schroder, M., 1995. Optimal portfolio selection with fixed transaction costs. Unpublished working paper. Northwestern University, Evanston, IL.

Stoll, H., Whaley, R., 1983. Transaction costs and the small firm effect. Journal of Financial Economics 12, 57-79. 
Tauchen, G., Hussey, R., 1991. Quadrature-based methods for obtaining approximate solutions to nonlinear asset pricing models. Econometrica 59, 317-96.

Vayanos, D., 1998. Transaction costs and asset prices: a dynamic equilibrium model. Review of Financial Studies, 1998, 11, 1-58. 
Table 1

Panel A: Unconditional sample moments and VAR coefficients

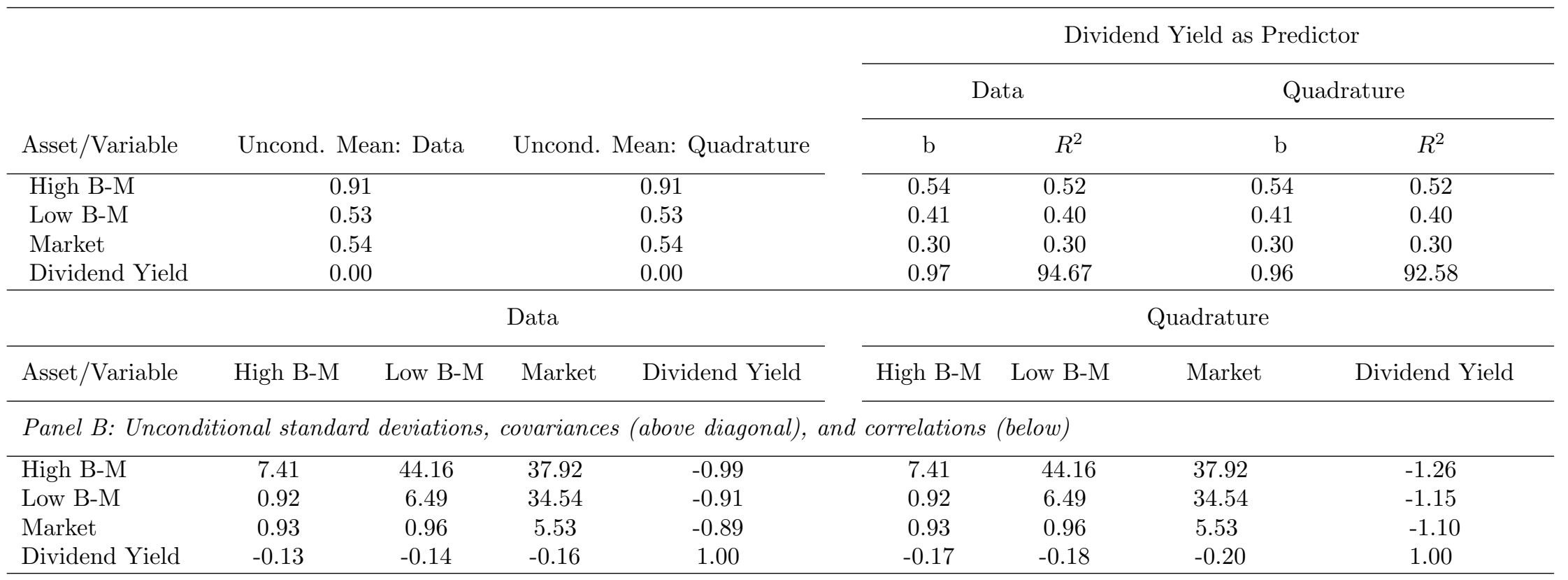

Panel C: Conditional standard deviations, covariances (above diagonal), and correlations (below) for the VAR with Dividend Yield as Predictor

\begin{tabular}{lccccccccc}
\hline High B-M & 7.39 & 43.94 & 37.75 & -1.51 & 7.39 & 43.94 & 37.75 \\
Low B-M & 0.92 & 6.48 & 34.41 & -1.31 & 0.92 & 6.48 & 34.41 & -1.77 & -1.54 \\
Market & 0.93 & 0.96 & 5.52 & -1.18 & 0.93 & 0.96 & -1.39 \\
Dividend Yield & -0.88 & -0.88 & -0.93 & 0.23 & -0.88 & -0.87 & -0.92
\end{tabular}

The table reports moments and parameters for high and low Book-to-Market (B-M) and market portfolios estimated from U.S. data and calculated for the quadrature approximation based on the VAR that uses log dividend yield as the only state variable. The data period is July 1927 to November 1996. Panel A reports unconditional sample moments for the data. Panel A also reports data and quadrature results for the two VARs: $\mathrm{b}$ is the vector of VAR slopes and $R^{2}$ denotes the regression $R^{2}$. Panel B reports the unconditional covariance matrix for the data and for the quadrature approximation. Panels C reports the conditional covariance matrices for the data VAR and the quadrature VAR using the $\log$ dividend yield as the state variable. All results are for continuously compounded returns. Returns are expressed per month and in percent. 
Table 2

\begin{tabular}{|c|c|c|c|c|c|c|c|c|c|c|}
\hline \multicolumn{11}{|c|}{ Utility Costs of Transactions Costs } \\
\hline & \multicolumn{2}{|c|}{ Market } & \multicolumn{2}{|c|}{ Low B-M } & \multicolumn{2}{|c|}{ High B-M } & \multicolumn{2}{|c|}{ Market \& Low B-M } & \multicolumn{2}{|c|}{ Market \& High B-M } \\
\hline & i.i.d. & Predict. & i.i.d. & Predict. & i.i.d. & Predict. & i.i.d. & Predict. & i.i.d. & Predict. \\
\hline \multirow[t]{2}{*}{ Proportional } & 0.096 & 0.477 & 0.138 & 0.730 & 0.252 & 0.904 & 0.096 & 0.656 & 0.252 & 0.905 \\
\hline & & $(0.037)$ & & $(0.043)$ & & $(0.094)$ & & $(0.006)$ & & $(0.096)$ \\
\hline \multirow[t]{2}{*}{ Proportional and Single Fixed } & 0.164 & 0.666 & 0.21 & 0.942 & 0.351 & 1.112 & 0.164 & 0.860 & 0.351 & 1.114 \\
\hline & & $(0.049)$ & & $(0.052)$ & & $(0.110)$ & & $(0.012)$ & & $(0.113)$ \\
\hline \multirow[t]{2}{*}{ Proportional and Fixed-for-each } & & & & & & & 0.164 & 0.863 & 0.351 & 1.114 \\
\hline & & & & & & & & $(0.012)$ & & $(0.112)$ \\
\hline
\end{tabular}

The table reports the percentage of wealth an investor would sacrifice to live in a no-transactions-costs-world when returns are i.i.d. (Panel A) and means and standard deviations of such percentage (Panel B) when returns are predictable. In each case, the dynamic investor problem is solved and the initial value of the inherited portfolio allocation is set equal to beginning-of-horizon portfolio allocation for the analogous dynamic problem with no transactions costs. Means and standard deviations are calculated using the unconditional distribution for the dividend yield. All values are reported in percent. Proportional transactions costs parameters are set at $\phi_{p}^{\mathrm{Market}}=0.25 \%, \phi_{p}^{\mathrm{High}} \mathrm{B}-\mathrm{M}=0.5 \%$, $\phi_{p}^{\text {Low B-M }}=0.375 \%$. Single Fixed sets the $\phi_{f}$ fixed cost parameters all equal to zero and $\phi_{F}=0.01 \%$ in equation (4) of the text. This means that a single $0.01 \%$ transactions cost is triggered if at least one asset is rebalanced. Fixed-for-each sets the fixed cost parameter $\phi_{f}$ for the market, the high B-M asset and the low B-M asset equal to $0.01 \%$ and sets $\phi_{F}=0 \%$ in equation (4) of the text. This means that an incremental $0.01 \%$ transactions costs will be triggered as each additional asset is rebalanced. The risk aversion parameter, $\gamma$, is fixed at 8 . 
Table 3

Utility Comparisons: One Risky Asset vs. the other

\begin{tabular}{lccc}
\hline & Market vs. High B-M & Low B-M vs. High B-M & Low B-M vs. Market \\
& Panel A: i.i.d. Returns & \\
\hline No Transactions Costs & 6.636 & 8.204 & 1.680 \\
Proportional & 6.490 & 8.103 & 1.722 \\
Proportional and Single Fixed & 6.462 & 8.084 & 1.734 \\
& Panel B: Predictable Returns & \\
& 13.485 & 14.624 & 1.302 \\
No Transactions Costs & $(1.680)$ & $(1.443)$ & $(0.265)$ \\
& 13.080 & 14.469 & 1.548 \\
Proportional & $(1.738)$ & $(1.492)$ & $(0.276)$ \\
Proportional and Single Fixed & 13.063 & 14.465 & 1.563 \\
& $(1.737)$ & $(1.504)$ & $(0.284)$
\end{tabular}

The table reports the percentage of wealth a investor would sacrifice to have the second risky asset rather than the first when returns are i.i.d. (Panel A) and means and standard deviations of such percentage (Panel B) when returns are predictable. In each case, the dynamic investor problem is solved and the initial value of the inherited portfolio allocation is set equal to beginning-of-horizon portfolio allocation for the analogous dynamic problem with no transactions costs. Means and standard deviations are calculated using the unconditional distribution for the dividend yield. All values are reported in percent. Proportional transactions costs parameters are set at $\phi_{p}^{\text {Market }}=0.25 \%, \phi_{p}^{\text {High B-M }}=0.5 \%, \phi_{p}^{\text {Low B-M }}=0.375 \%$. Single Fixed sets the $\phi_{f}$ fixed cost parameters all equal to zero and $\phi_{F}=0.01 \%$ in equation (4) of the text. This means that a single $0.01 \%$ transactions cost is triggered if the risky asset is rebalanced. The risk aversion parameter, $\gamma$, is fixed at 8 . 
Utility Costs of Restricted Asset Access: One Asset vs. Two Assets

Market vs. Market \& High High vs. Market \& High Market vs. Market\& Low Low vs. Market \& Low

Panel A: i.i.d. Returns

\begin{tabular}{|c|c|c|c|c|}
\hline No Transactions Costs & 6.636 & 0.000 & 0.000 & 1.680 \\
\hline Proportional & 6.490 & 0.000 & 0.000 & 1.722 \\
\hline Proportional and Single Fixed & 6.462 & 0.000 & 0.000 & 1.734 \\
\hline Proportional and Fixed-for-each & 6.462 & 0.000 & 0.000 & 1.734 \\
\hline \multicolumn{5}{|c|}{ Panel B: Predictable Returns } \\
\hline No Transactions Costs & $\begin{array}{l}13.503 \\
(1.680)\end{array}$ & $\begin{array}{c}0.003 \\
(0.002)\end{array}$ & $\begin{array}{c}0.438 \\
(0.102)\end{array}$ & $\begin{array}{c}1.730 \\
(0.171)\end{array}$ \\
\hline Proportional & $\begin{array}{l}13.086 \\
(1.720)\end{array}$ & $\begin{array}{c}0.004 \\
(0.001)\end{array}$ & $\begin{array}{c}0.256 \\
(0.065)\end{array}$ & $\begin{array}{c}1.813 \\
(0.204)\end{array}$ \\
\hline Proportional and Single Fixed & $\begin{array}{c}13.067 \\
(1.723)\end{array}$ & $\begin{array}{c}0.004 \\
(0.001)\end{array}$ & $\begin{array}{c}0.244 \\
(0.062)\end{array}$ & $\begin{array}{c}1.824 \\
(0.211)\end{array}$ \\
\hline Proportional and Fixed-for-each & $\begin{array}{l}13.065 \\
(1.722)\end{array}$ & $\begin{array}{c}0.004 \\
(0.001)\end{array}$ & $\begin{array}{c}0.243 \\
(0.064)\end{array}$ & $\begin{array}{c}1.823 \\
(0.213)\end{array}$ \\
\hline
\end{tabular}

The table reports the percentage of wealth an investor would sacrifice to gain access to a second risky asset when returns are i.i.d. (Panel A) and means and standard deviations of such percentage (Panel B) when returns are predictable. In each case, the dynamic investor problem is solved and the initial value of the inherited portfolio allocation is set equal to beginning-of-horizon portfolio allocation for the analogous dynamic problem with no transactions costs. Means and standard deviations are calculated using the unconditional distribution for the dividend yield. All values are reported in percent. Proportional transactions costs parameters are set at $\phi_{p}^{\text {Market }}=0.25 \%$, $\phi_{p}^{\text {High B-M }}=0.5 \%, \phi_{p}^{\text {Low B-M }}=0.375 \%$. Single Fixed sets the $\phi_{f}$ fixed cost parameters all equal to zero and $\phi_{F}=0.01 \%$ in equation $(4)$ of the text. This means that a single $0.01 \%$ transactions cost is triggered if at least one asset is rebalanced. Fixed-for-each sets the fixed cost parameter $\phi_{f}$ for the market, the high B-M asset and the low $\mathrm{B}-\mathrm{M}$ asset equal to $0.01 \%$ and sets $\phi_{F}=0 \%$ in equation (4) of the text. This means that an incremental $0.01 \%$ transactions costs will be triggered as each additional asset is rebalanced. The risk aversion parameter, $\gamma$, is fixed at 8 . 
Table 5

Utility Costs of Ignoring Risky Asset Return Predictability

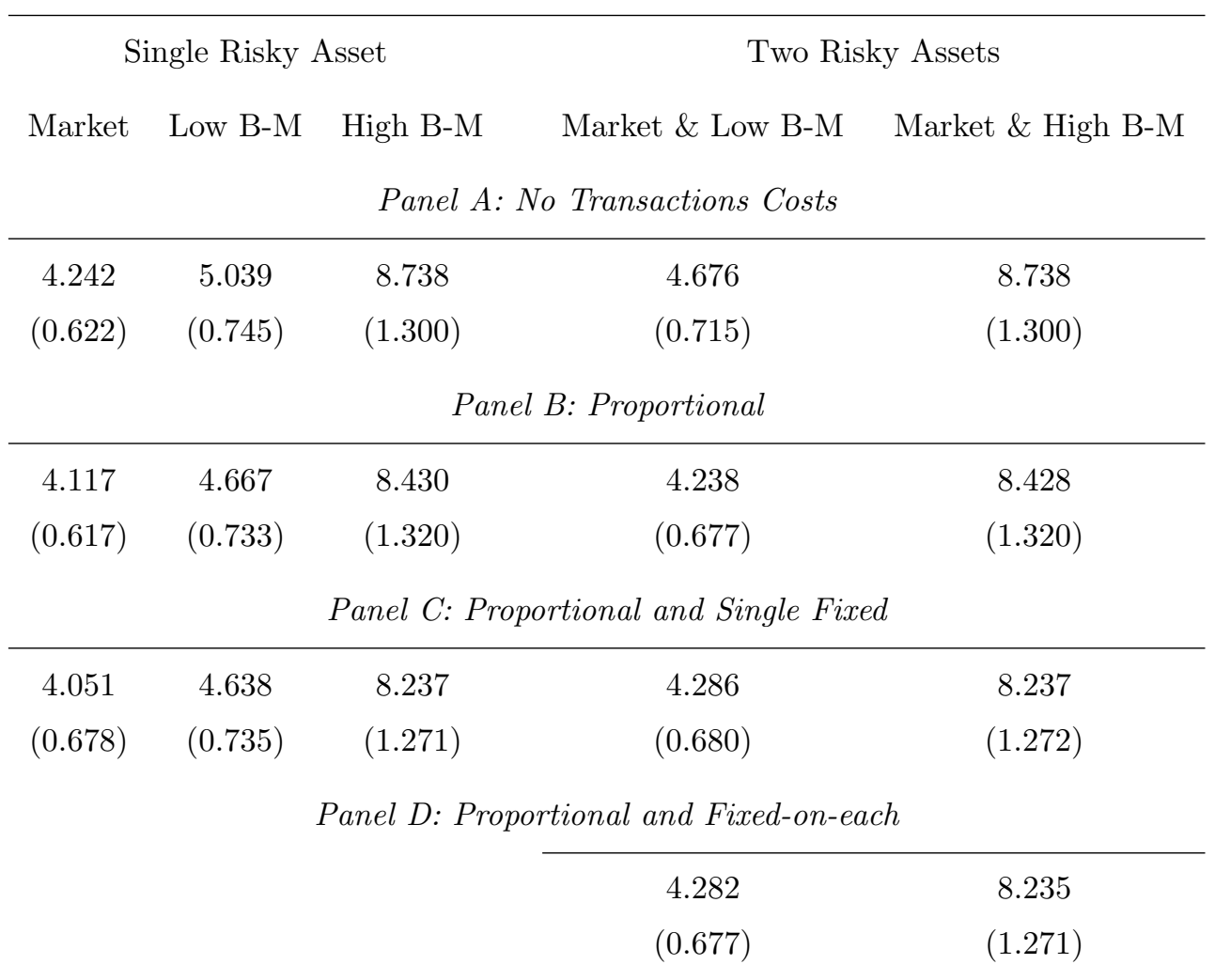

The table reports the mean and standard deviation (in parenthesis) of the percentage of wealth an investor would sacrifice to gain access to predictive information for risky asset returns. First, the dynamic investor problem is solved using the unconditional return distribution at each iteration through the 20-year investment horizon. Second, optimal policies from this solution are fed into an otherwise identical problem with return predictability. In each case and for each of the 19 dividend states, the initial value of the inherited portfolio allocation is set equal to beginning-of-horizon portfolio allocation for the analogous dynamic problem with i.i.d. returns and no transactions costs. Means and standard deviations are calculated using the unconditional distribution for the dividend yield. All values are reported in percent. Proportional transactions costs parameters are set at $\phi_{p}^{\text {Market }}=0.25 \%, \phi_{p}^{\text {High B-M }}=0.5 \%, \phi_{p}^{\text {Low B-M }}=0.375 \%$. Single Fixed sets the $\phi_{f}$ fixed cost parameters all equal to zero and $\phi_{F}=0.01 \%$ in equation (4) of the text. This means that a single $0.01 \%$ transactions cost is triggered if at least one asset is rebalanced. Fixed-for-each sets the fixed cost parameter $\phi_{f}$ for the market, the high B-M asset and the low B-M asset equal to $0.01 \%$ and sets $\phi_{F}=0 \%$ in equation (4) of the text. This means that an incremental $0.01 \%$ transactions costs will be triggered as each additional asset is rebalanced. The risk aversion parameter, $\gamma$, is fixed at 8 . 


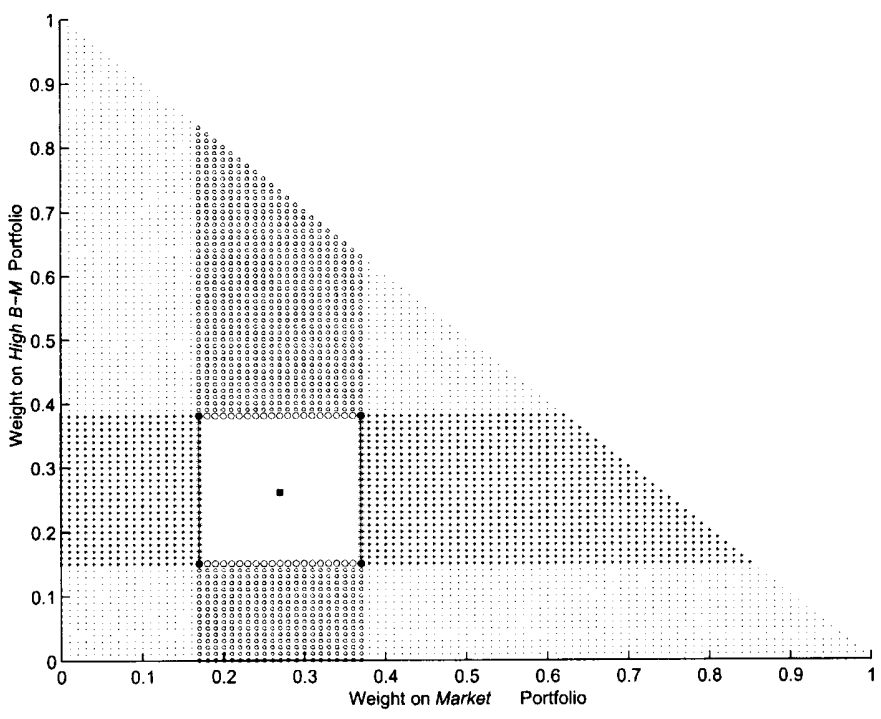

1.a $\rho=0$

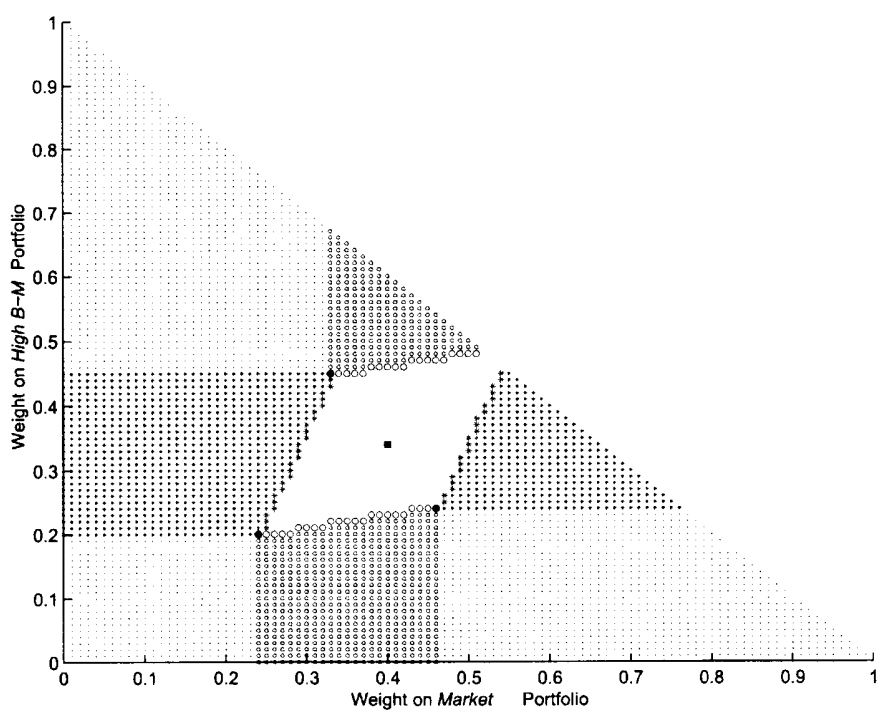

1.c $\rho=-30 \%$ of data

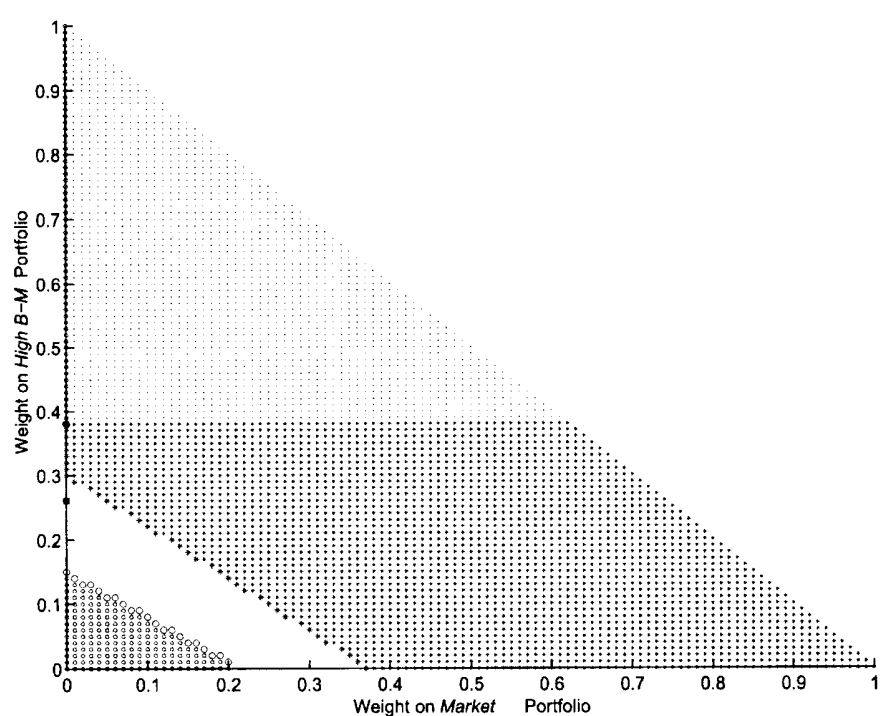

1.b $\rho$ as in data

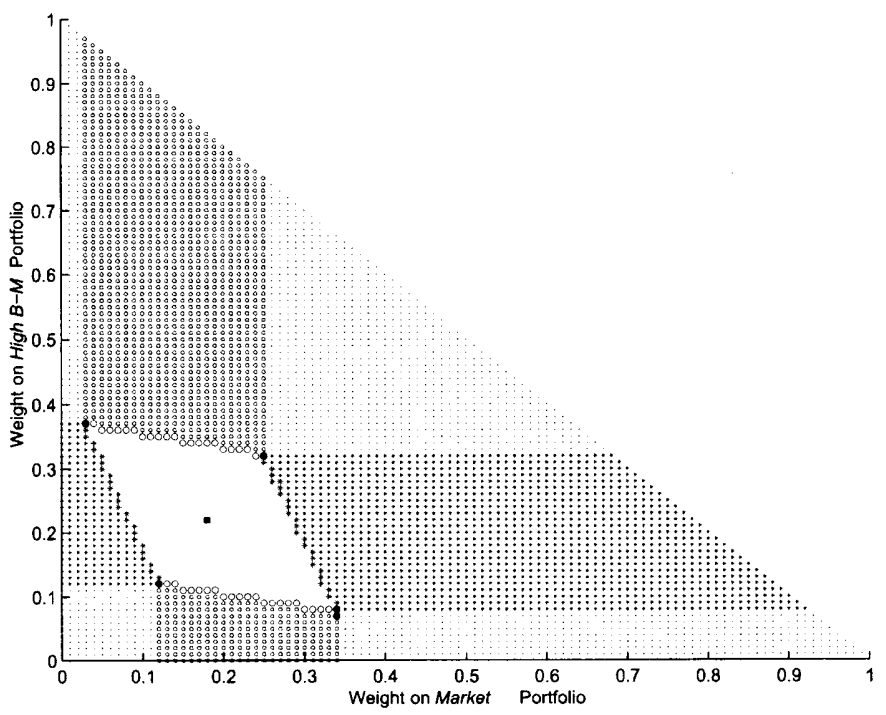

$1 . \mathrm{d} \rho=30 \%$ of data

Figure 1. Rebalancing rules with proportional costs: i.i.d. returns. The two risky assets available are a portfolio of high book-to-market stocks (high B-M asset) and the market. Each blank region and its larger-marker boundary represent the allocation states in which the agent does not trade. Larger markers represent target portfolios for the states represented by the analogous smaller markers. Each allocation state marked by a smaller solid dot rebalances to the closest larger solid dot: both weights change with such rebalancing. Each allocation state marked by a smaller cross (circle) rebalances horizontally (vertically) to the corresponding larger cross (circle): only the weight on the market (high B-M asset) changes with such rebalancing. $\rho$ stands for the unconditional correlation between the assets. Subfigures a, b, c, d ( e, f, g, h) scale correlations as labelled and report for the last (first) month of the 20-year investment horizon. In each case, the solid square represents the unique target portfolio in the associated no-transactions-costs world. Log returns are i.i.d. normal and the agent is a dynamic optimizer. A standard Gaussian quadrature rule is used to discretize returns. The calibration is based on historical data from $7 / 27$ to $11 / 96$. The risk aversion parameter, $\gamma$, is fixed at 8 . We set proportional cost parameters $\phi_{p}^{\text {Market }}=0.25 \%$ and $\phi_{p}^{\text {High B-M }}=0.5 \%$ and all other parameters to zero in equation (4) of the text. 


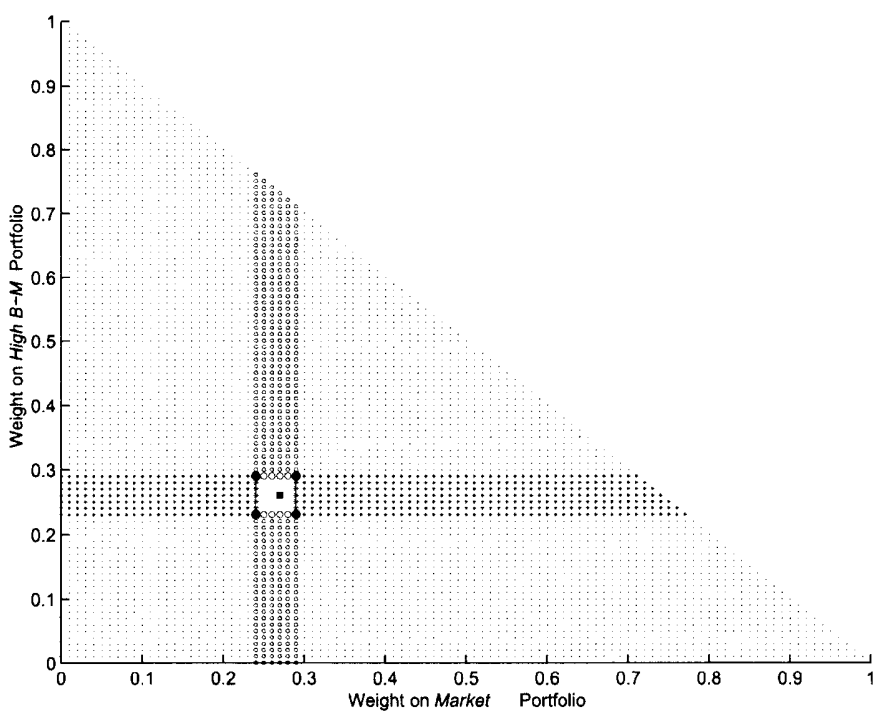

1.e $\rho=0$

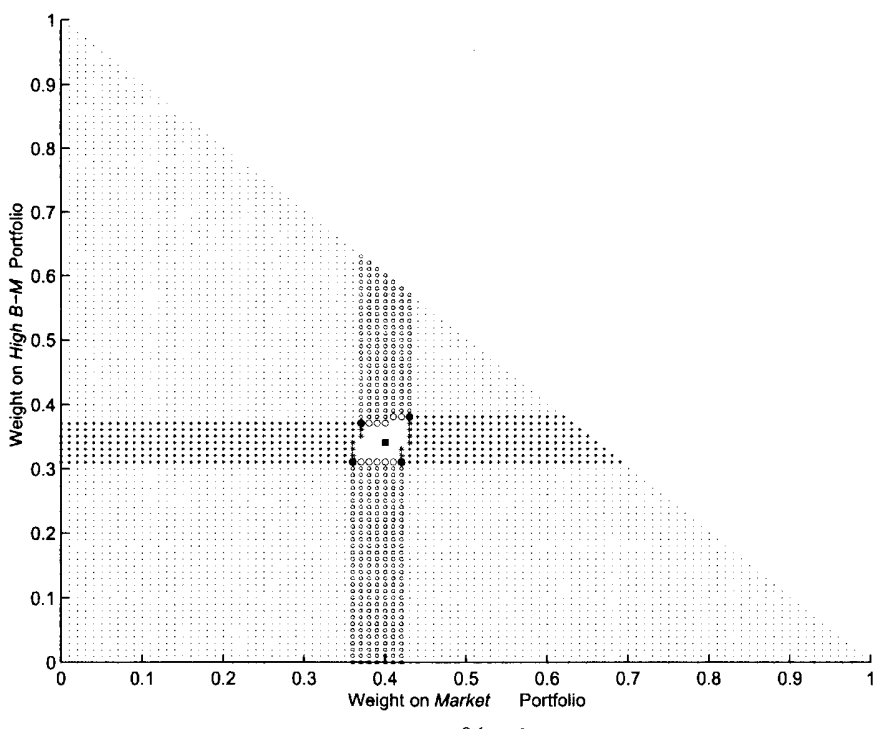

1.g $\rho=-30 \%$ of data

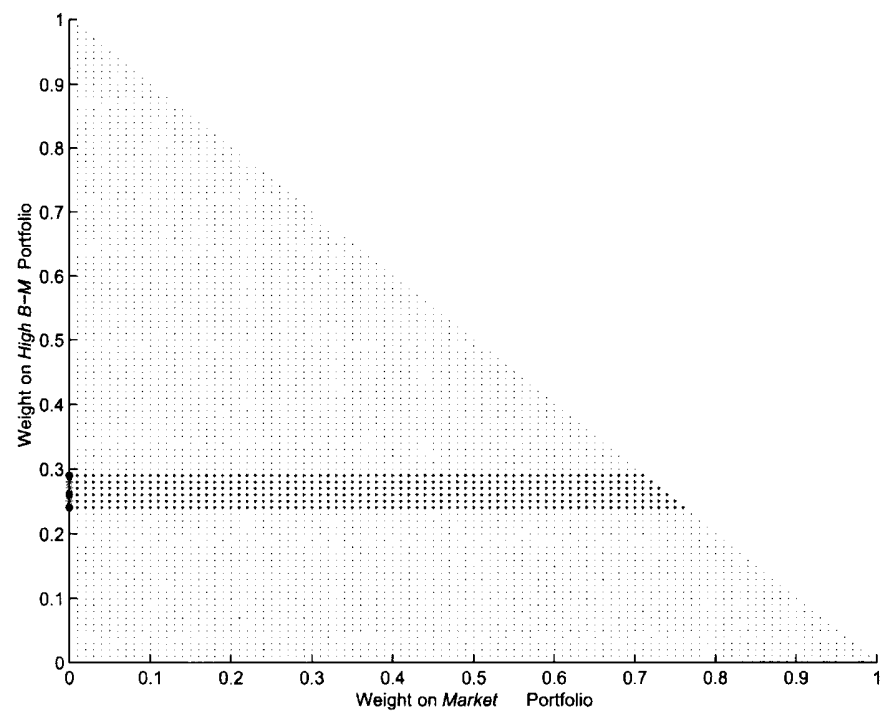

1.f $\rho$ as in data

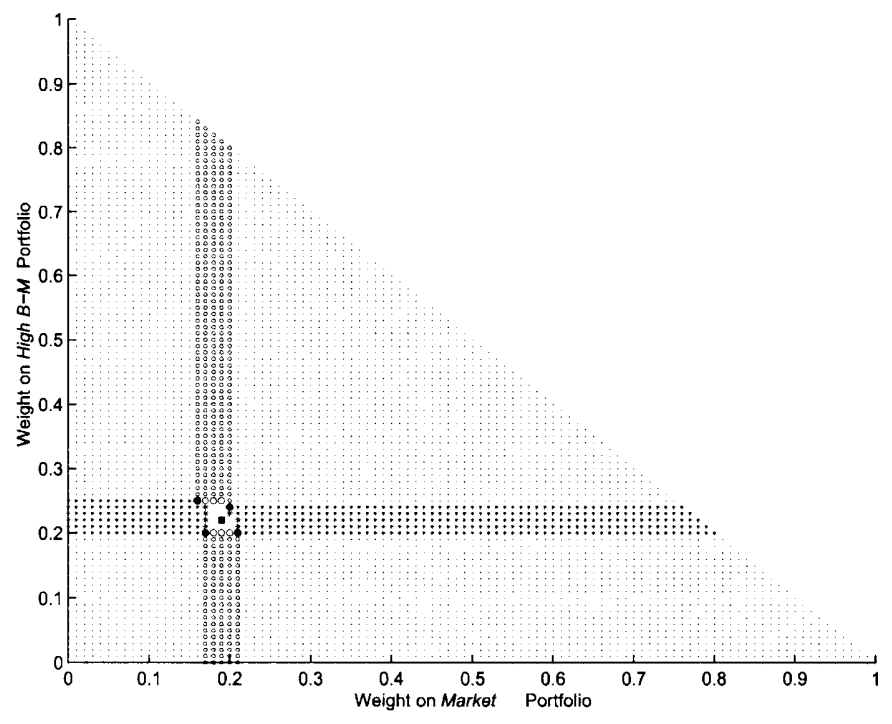

1.h $\rho=30 \%$ of data

Figure 1. Rebalancing rules with proportional costs: i.i.d. returns. (cont'd.) 

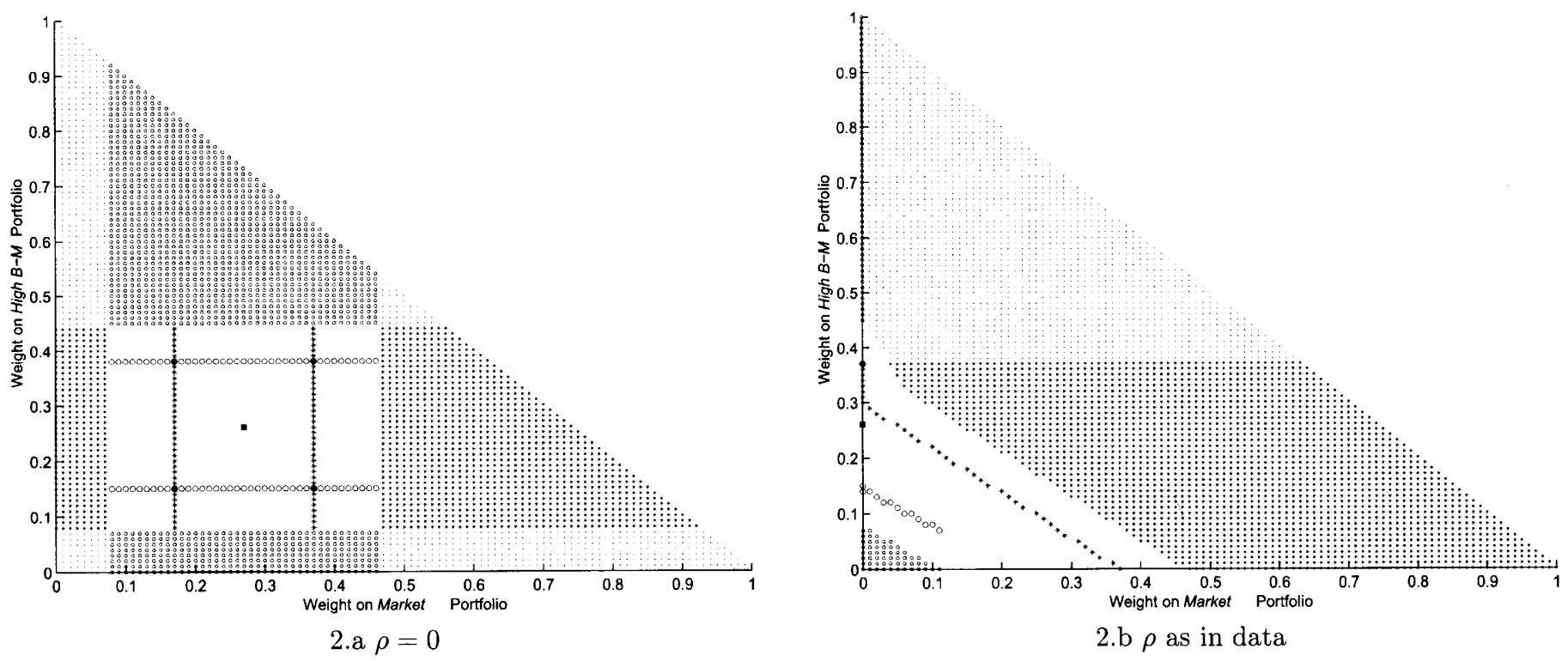

2.b $\rho$ as in data
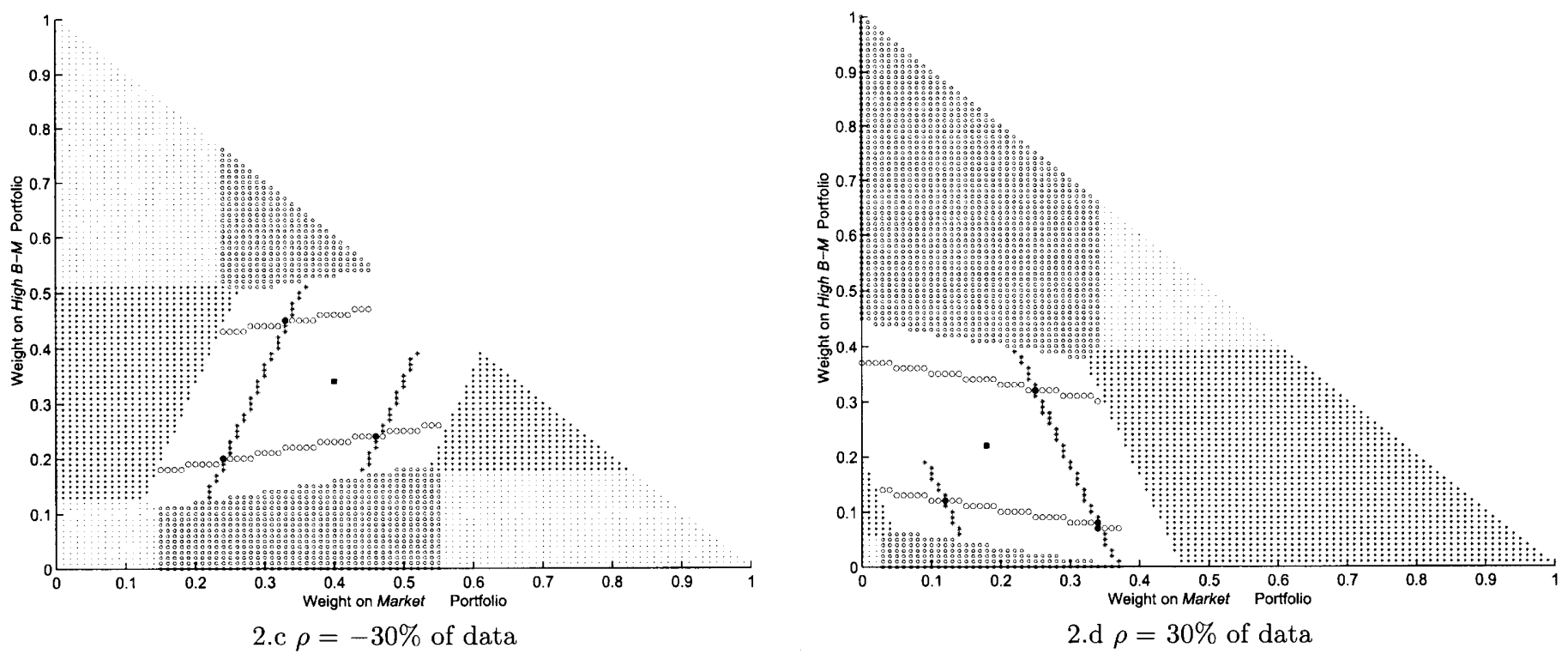

Figure 2. Rebalancing rules with proportional and fixed costs for each asset: i.i.d. returns. The two risky assets available are a portfolio of high book-to-market stocks (high B-M asset) and the market. Each blank region and larger-marker lines inside represent the allocation states in which the agent does not trade. Larger markers represent target portfolios for the states represented by the analogous smaller markers. Each allocation state marked by a smaller solid dot rebalances to the closest larger solid dot: both weights change with such rebalancing. Each allocation state marked by a smaller cross (circle) rebalances horizontally (vertically) to the corresponding larger cross (circle): only the weight on the market (high B-M asset) changes with such rebalancing. $\rho$ stands for the unconditional correlation between the assets. Subfigures a, b, c, d (e, f, g, h) scale correlations as labelled and report for the last (first) month of the 20-year investment horizon. In each case, the solid square represents the unique target portfolio in the associated no-transactions-costs world. Log returns are i.i.d. normal and the agent is a dynamic optimizer. A standard Gaussian quadrature rule is used to discretize returns. The calibration is based on historical data from $7 / 27$ to $11 / 96$. The risk aversion parameter, $\gamma$, is fixed at 8. We set proportional cost parameters $\phi_{p}{ }^{\text {Market }}=0.25 \%$ and $\phi_{p}{ }^{\text {High B-M }}=0.5 \%$ and fixed cost parameters $\phi_{f}^{\text {Market }}=0.01 \%$ and $\phi_{f}^{\text {High B-M }}=0.01 \%$ and $\phi_{F}=0 \%$ in equation (4) of the text. 

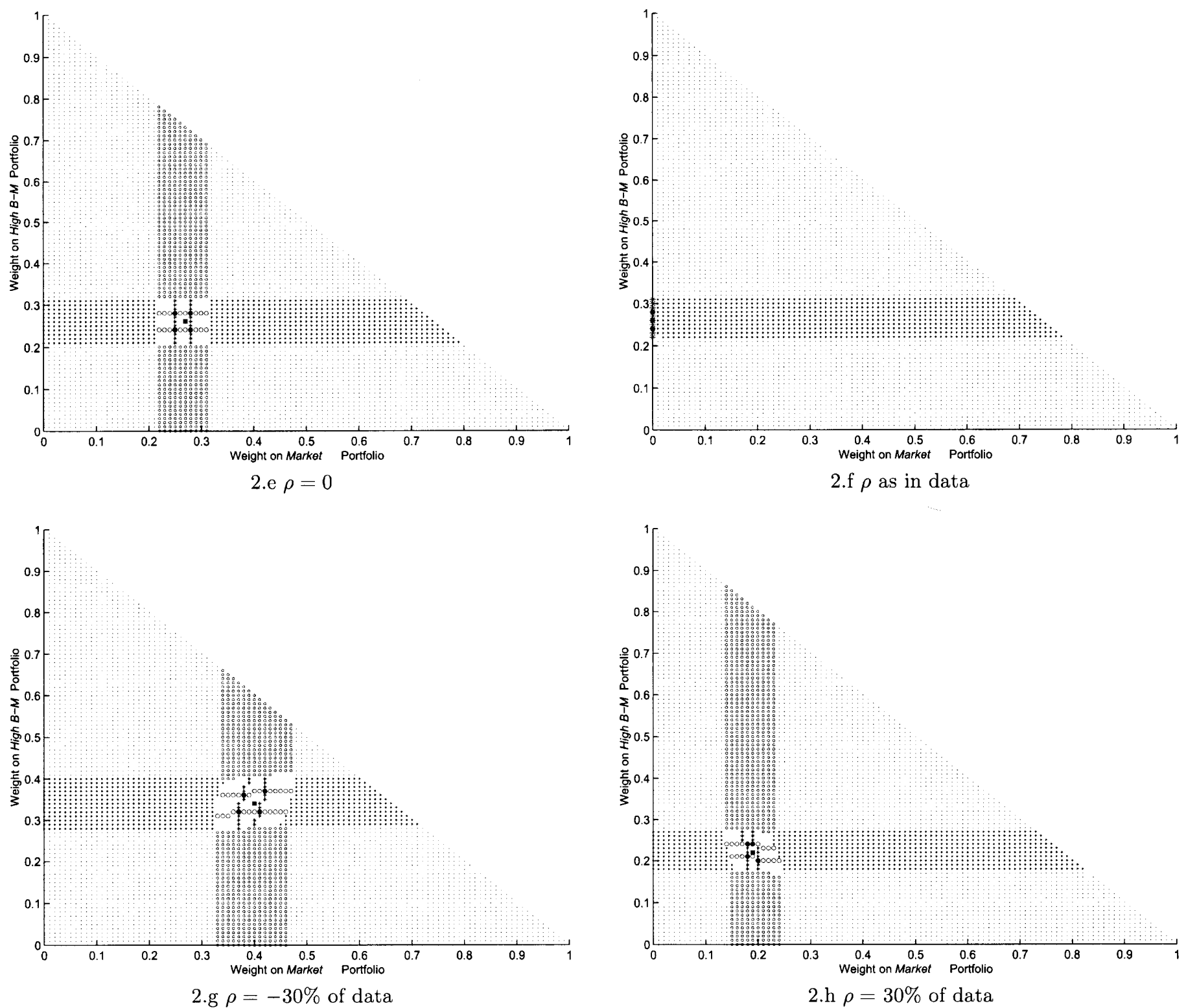

Figure 2. Rebalancing rules with proportional and fixed costs for each asset: i.i.d. returns. (cont'd.) 


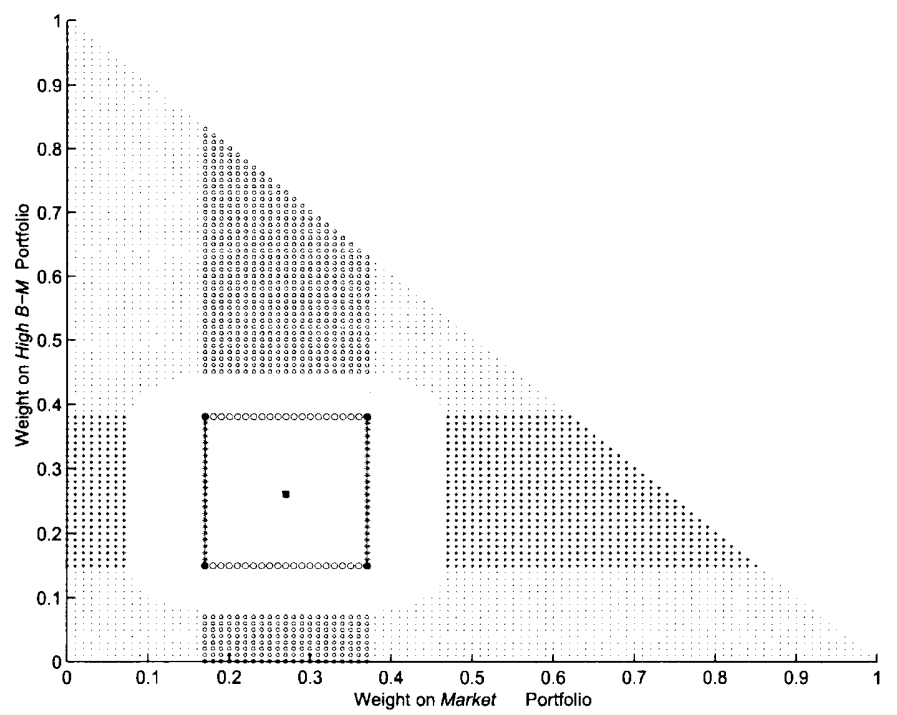

3.a $\rho=0$

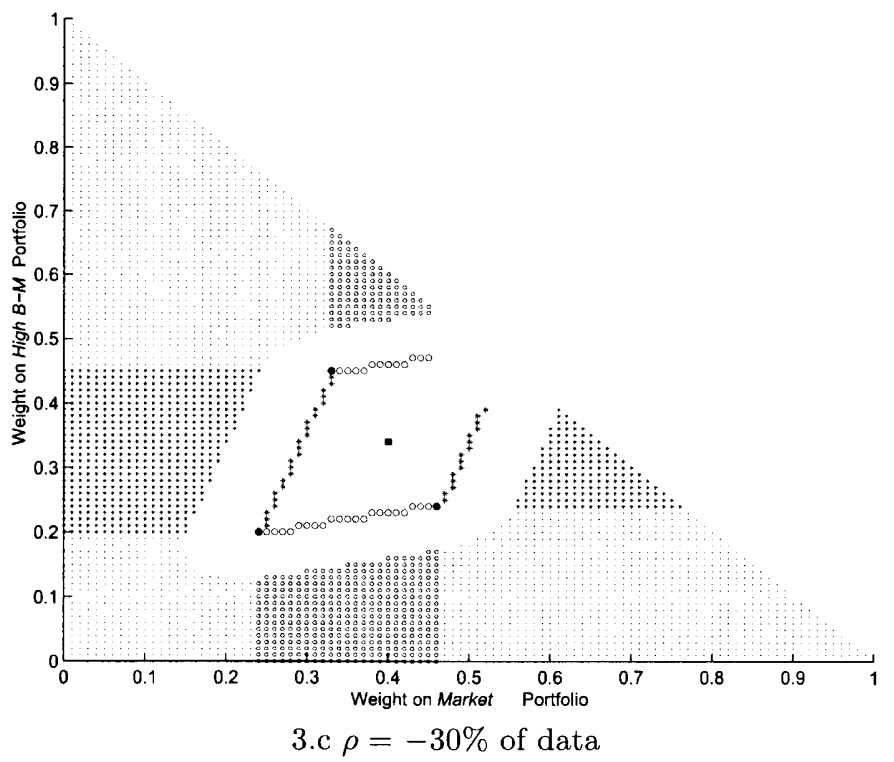

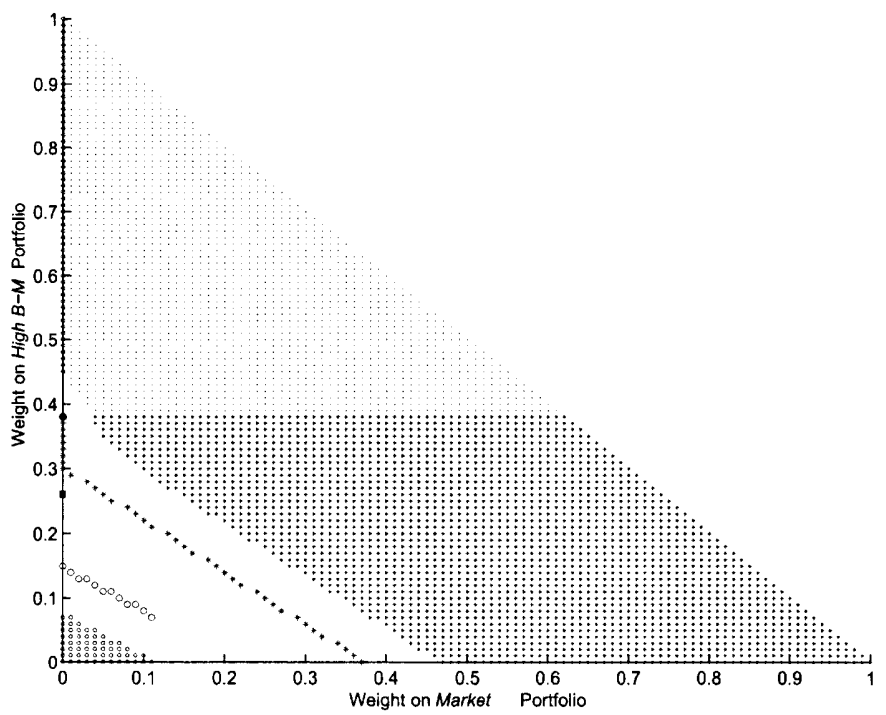

3.b $\rho$ as in data

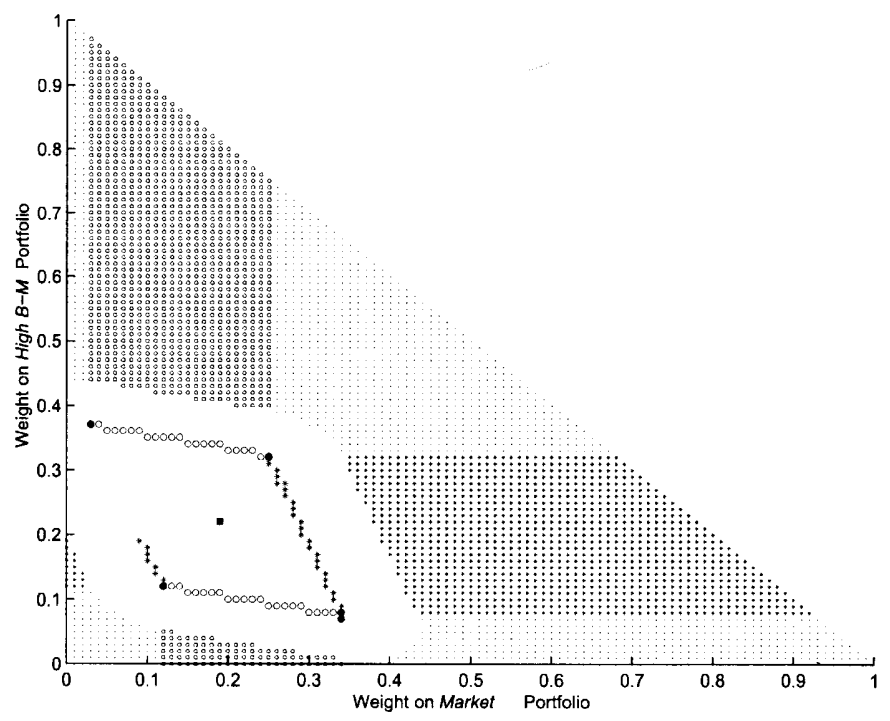

3.d $\rho=30 \%$ of data

Figure 3. Rebalancing rules with proportional and single fixed cost : i.i.d. returns. The two risky assets available are a portfolio of high book-to-market stocks (high B-M asset) and the market. Each blank region and larger-marker lines inside represent the allocation states in which the agent does not trade. Larger markers represent target portfolios for the states represented by the analogous smaller markers. Each allocation state marked by a smaller solid dot rebalances to the closest larger solid dot: both weights change with such rebalancing. Each allocation state marked by a smaller cross (circle) rebalances horizontally (vertically) to the corresponding larger cross (circle): only the weight on the market (high B-M asset)changes with such rebalancing. $\rho$ stands for the unconditional correlation between the assets. Subfigures a, b, c, d (e, f, g, h) scale correlations as labelled and report for the last (first) month of the 20-year investment horizon. In each case, the solid square represents the unique target portfolio in the associated no-transactions-costs world. Log returns are i.i.d. normal and the agent is a dynamic optimizer. A standard Gaussian quadrature rule is used to discretize returns. The calibration is based on historical data from $7 / 27$ to $11 / 96$. The risk aversion parameter, $\gamma$, is fixed at 8 . We set proportional cost parameters $\phi_{p}^{\text {Market }}=0.25 \%$ and $\phi_{p}{ }^{\text {High B-M }}=0.5 \%$ and the fixed cost parameter $\phi_{\mathrm{F}}=0.01 \%$ parameters to zero in equation (4) of the text. 


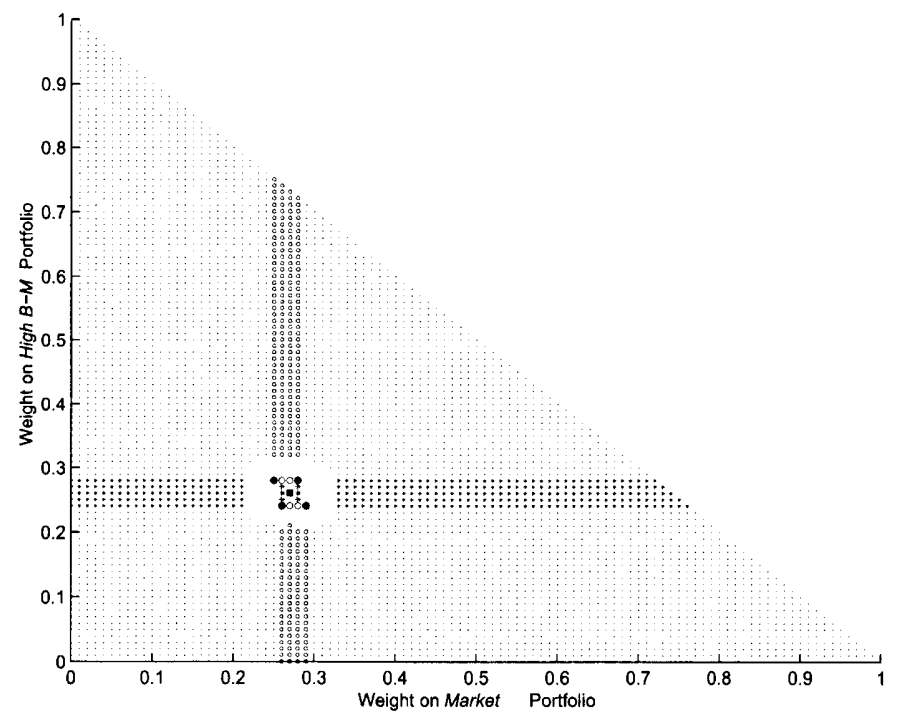

3.e $\rho=0$

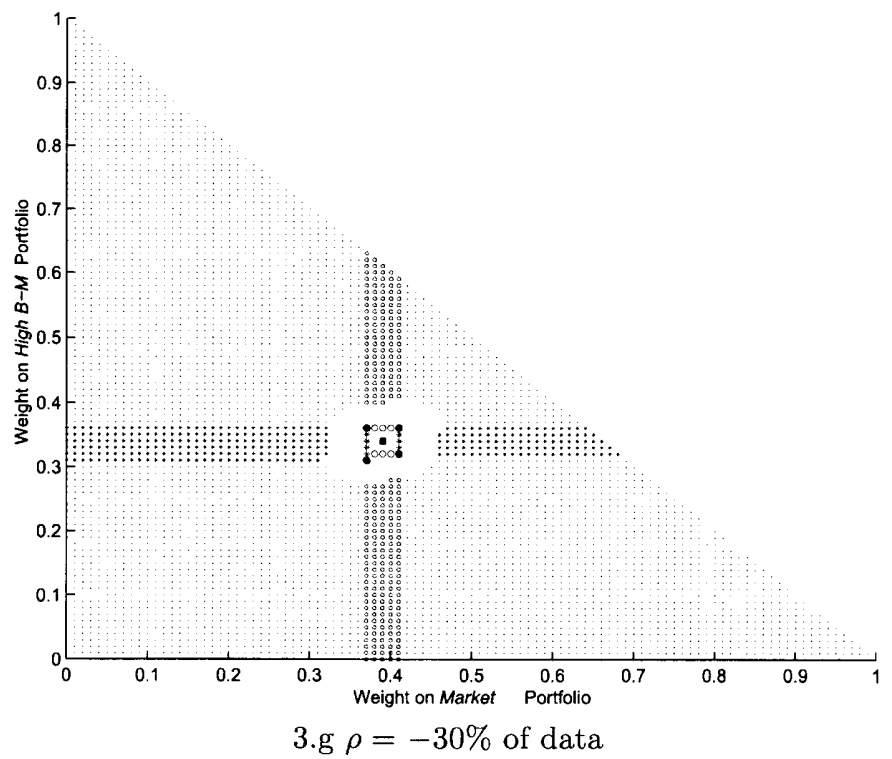

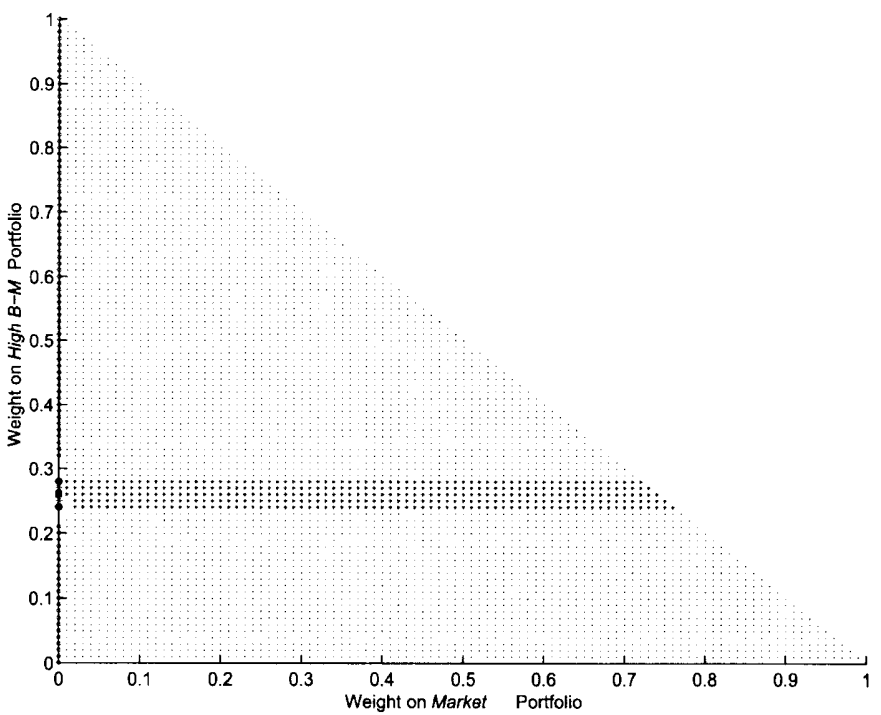

3.f $\rho$ as in data

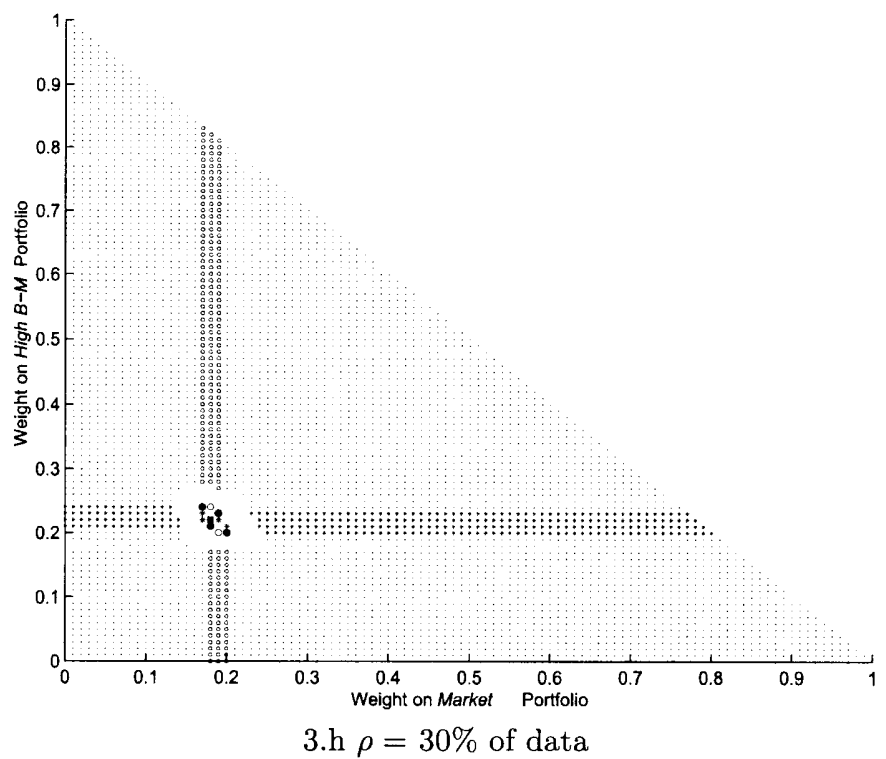

Figure 3. Rebalancing rules with proportional and single fixed cost : i.i.d. returns. (cont'd.) 


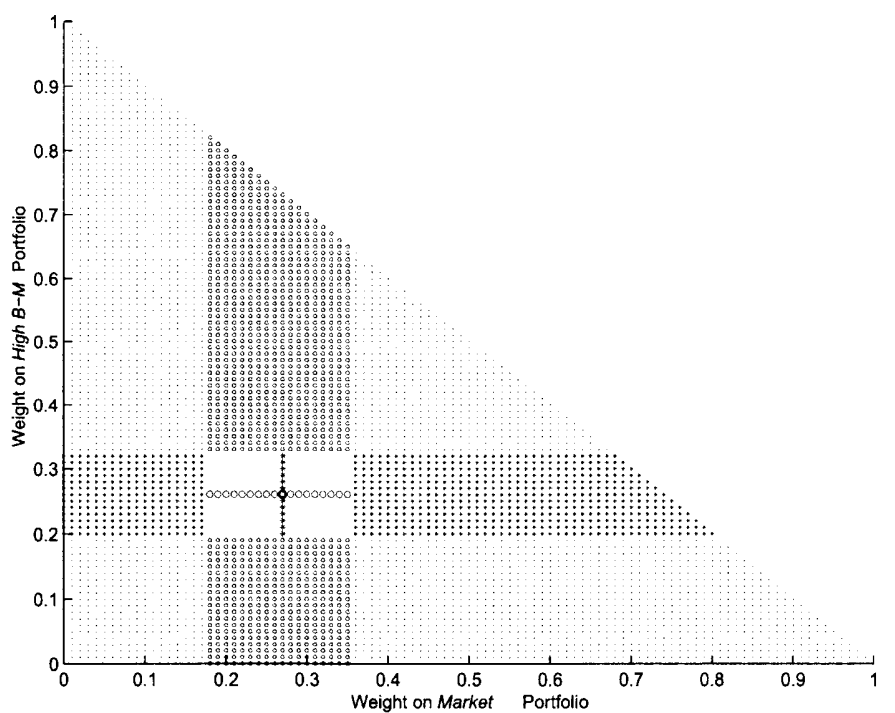

4.a $\rho=0$

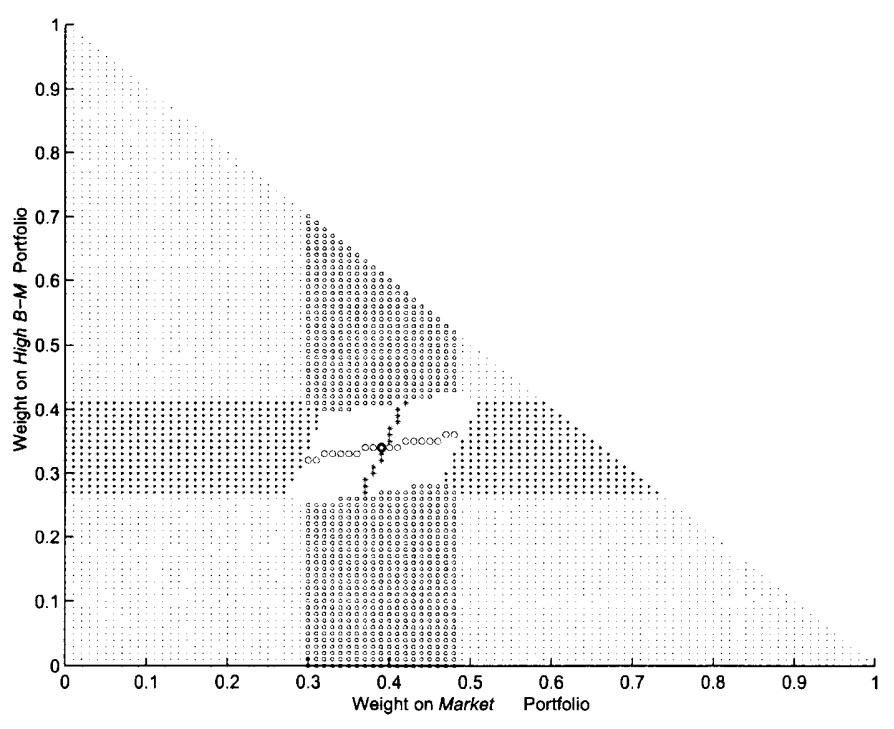

4.c $\rho=-30 \%$ of data

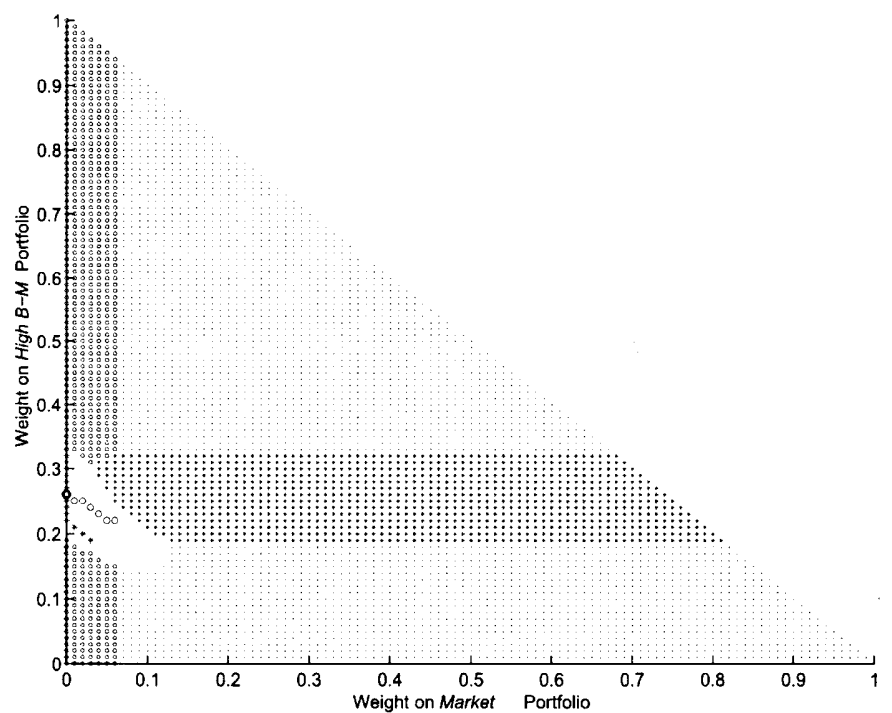

4.b $\rho$ as in data

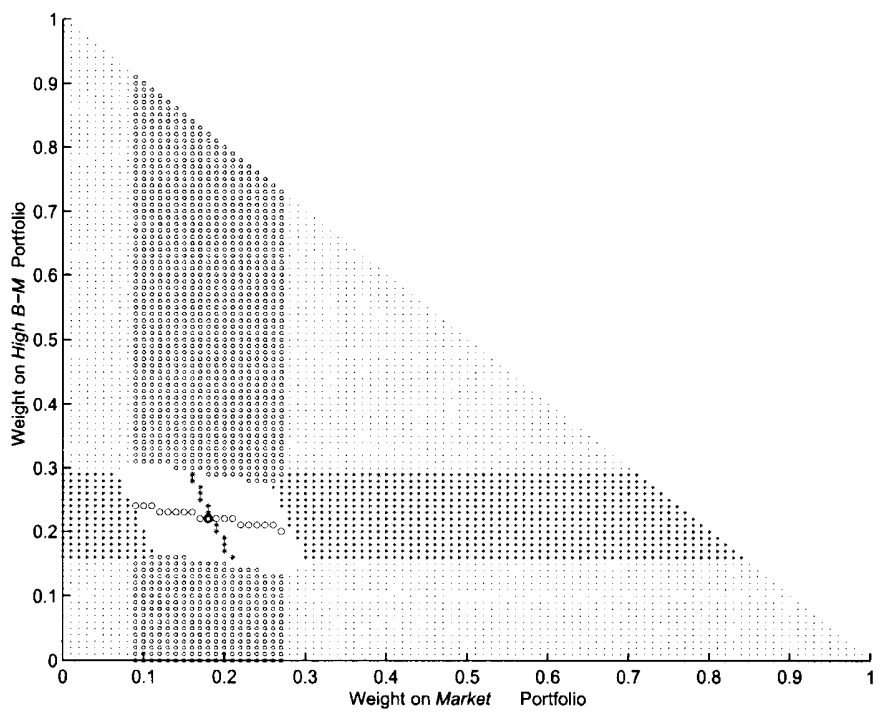

4.d $\rho=30 \%$ of data

Figure 4. Rebalancing rules with fixed costs for each asset: i.i.d. returns. The two risky assets available are a portfolio of high book-to-market stocks (high B-M asset) and the market. Each blank region and larger-marker lines inside represent the allocation states in which the agent does not trade. Larger markers represent target portfolios for the states represented by the analogous smaller markers. Each allocation state marked by a smaller solid dot rebalances to the closest larger solid dot: both weights change with such rebalancing. Each allocation state marked by a smaller cross (circle) rebalances horizontally (vertically) to the corresponding larger cross(circle): only the weight on the market (high B-M asset) changes with such rebalancing. $\rho$ stands for the unconditional correlation between the assets. Subfigures a, b, c, d (e, f, g, h) scale correlations as labelled and report for the last (first) month of the 20-year investment horizon. In each case, the unique target portfolio in the associated no-transactionscosts world coincides with the unique target portfolio for the allocation states represented by smaller solid dots in the transactions-costs world. These portfolios are represented by solid circles with empty squares inside. Log returns are i.i.d. normal and the agent is a dynamic optimizer. A standard Gaussian quadrature rule is used to discretize returns. The calibration is based on historical data from $7 / 27$ to $11 / 96$. Risk aversion parameter, $\gamma$, is fixed at 8 . We set fixed cost parameters $\phi_{f}^{\text {Market }}=0.01 \%$ and $\phi_{f}^{\text {High B-M }}=0.01 \%$ and all other parameters to zero in equation (4) of the text. 


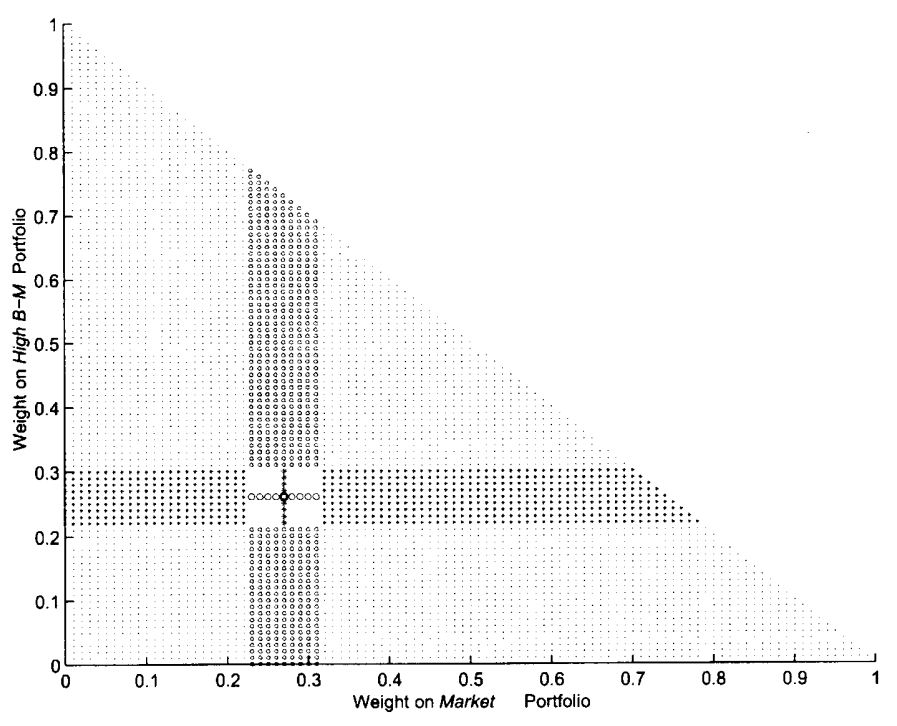

4.e $\rho=0$

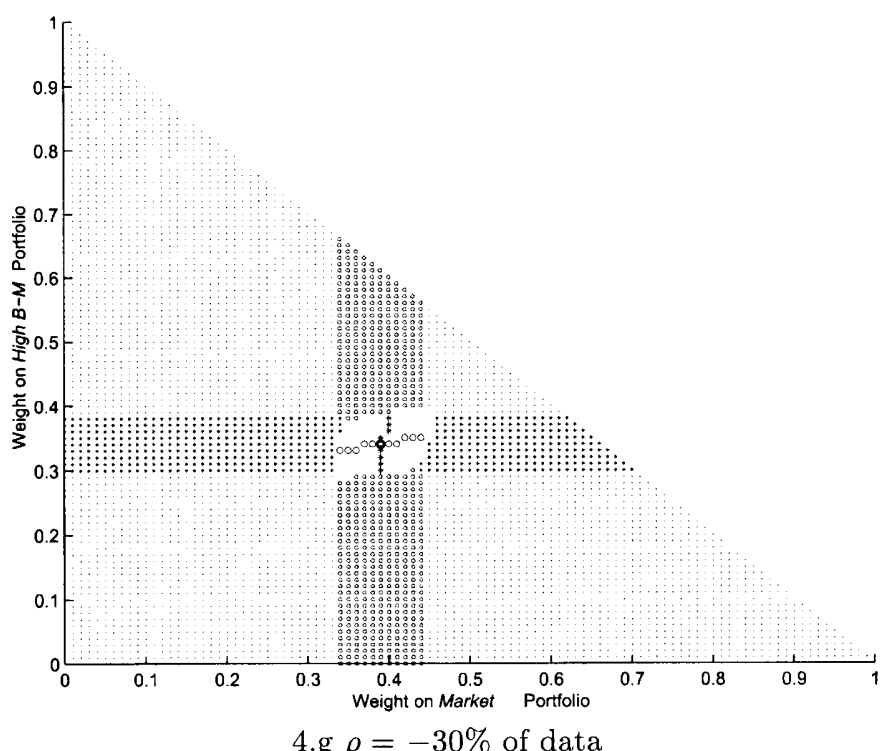

4.g $\rho=-30 \%$ of data
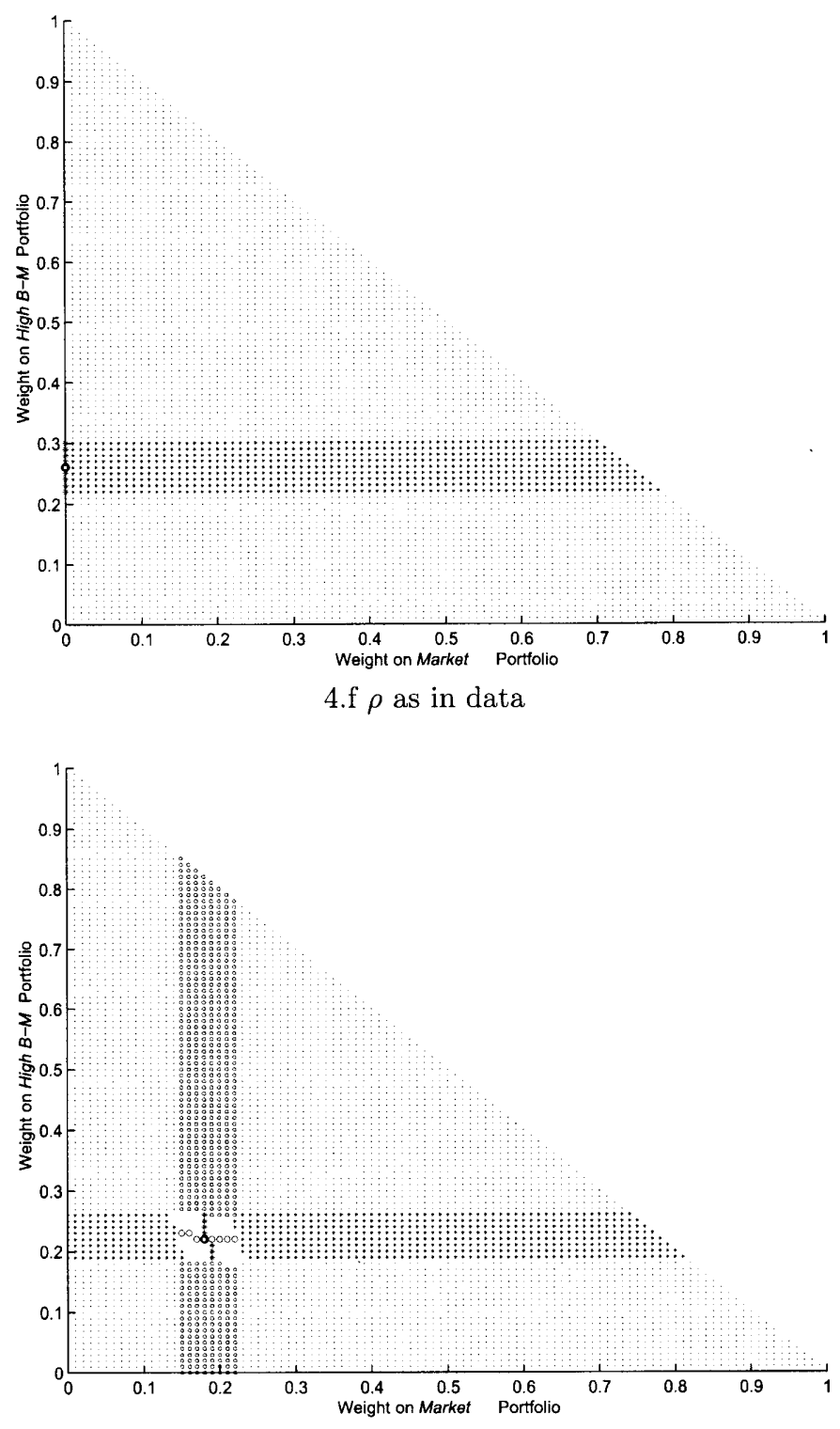

4.h $\rho=30 \%$ of data

Figure 4. Rebalancing rules with fixed costs for each asset: i.i.d. returns. (cont'd.) 


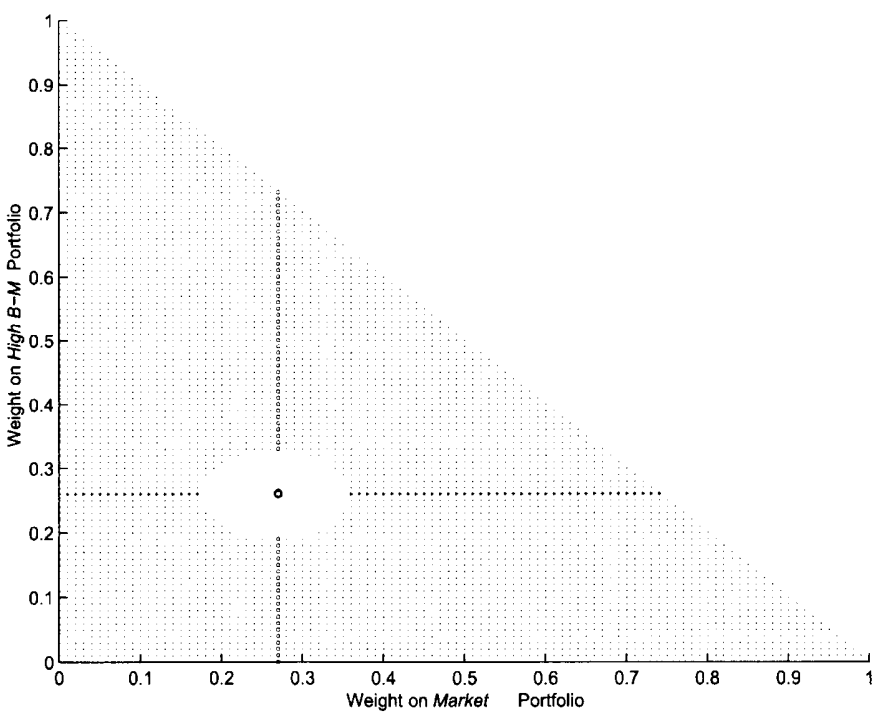

5.a $\rho=0$

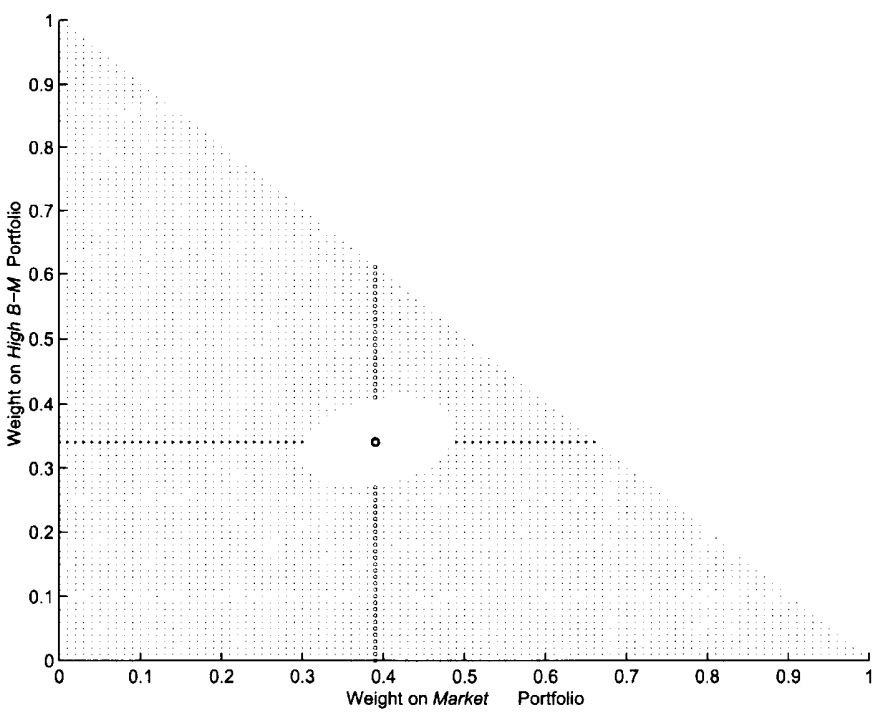

5.c $\rho=-30 \%$ of data

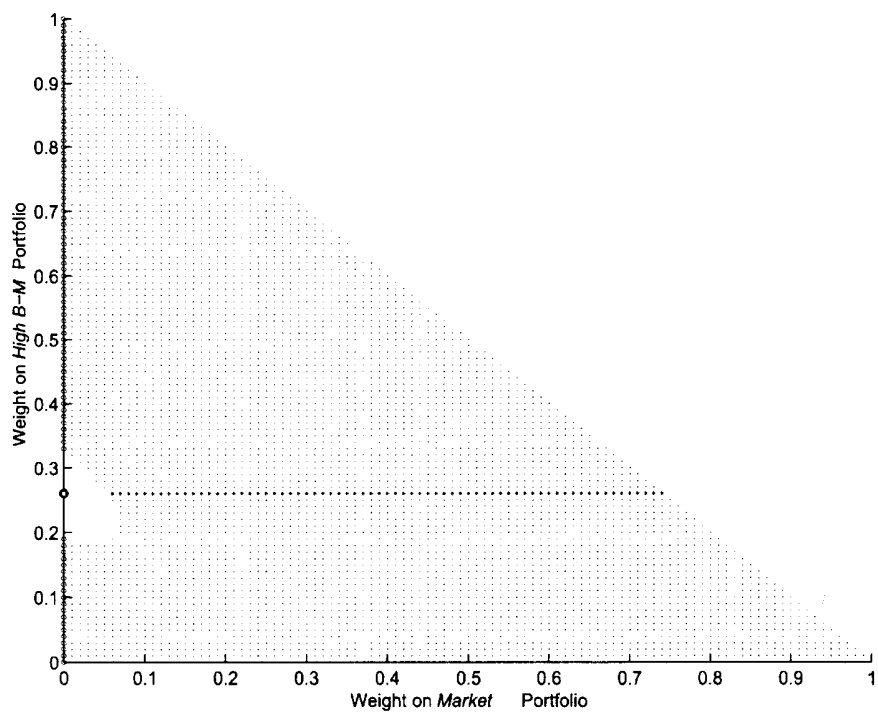

5.b $\rho$ as in data

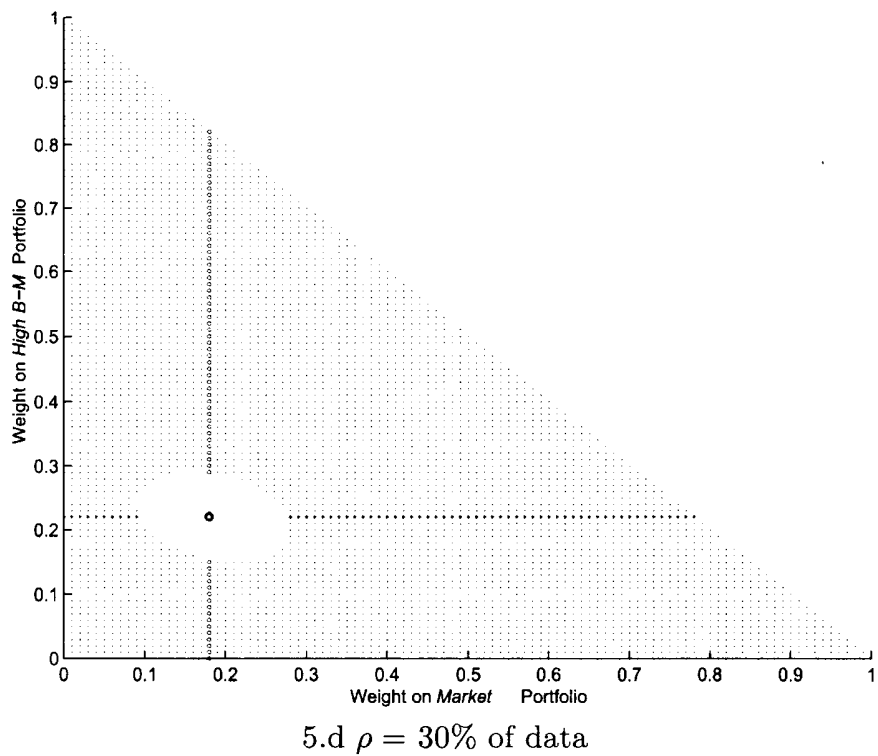

Figure 5. Rebalancing rules with single fixed cost: i.i.d. returns. The two risky assets available are a portfolio of high book-to-market stocks (high B-M asset) and the market. Each blank region with a single marker point inside represents the allocation states in which the agent does not trade. In each case, the unique target portfolio in the associated no-transactions-costs world coincides with the unique target portfolio in the transactions-costs world. These portfolios are represented by solid circles with empty squares inside. $\rho$ stands for the unconditional correlation between the assets. Subfigures a, b, c, d (e, f, g, h) scale correlations as labelled and report for the last (first) month of the 20-year investment horizon. For sake of consistency allocation states that horizontally or vertically rebalance to the unique optima are also marked. Log returns are i.i.d. normal and the agent is a dynamic optimizer. A standard Gaussian quadrature rule is used to discretize returns. The calibration is based on historical data from $7 / 27$ to $11 / 96$. Risk aversion parameter, $\gamma$, is fixed at 8 . We set the fixed cost parameter $\phi_{F}=0.01 \%$ and all other parameters to zero in equation (4) of the text. 

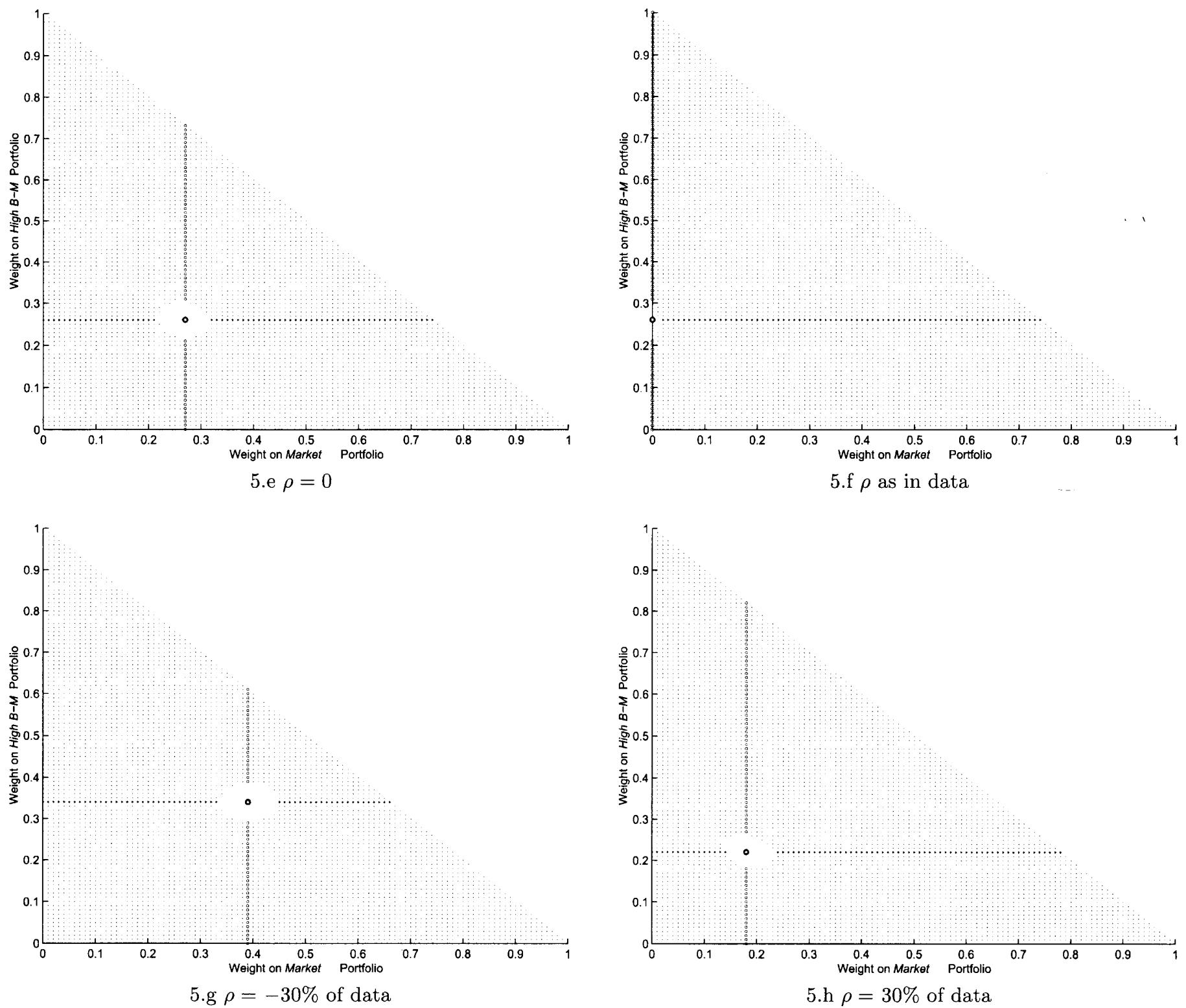

Figure 5. Rebalancing rules with single fixed cost: i.i.d. returns. (cont'd.) 


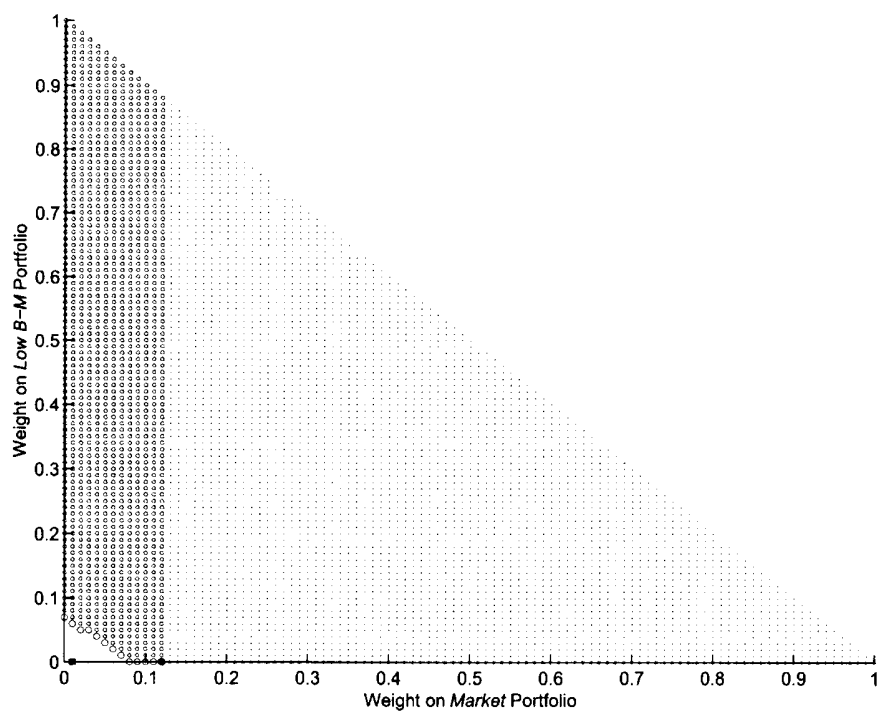

6.a dividend state $=1$

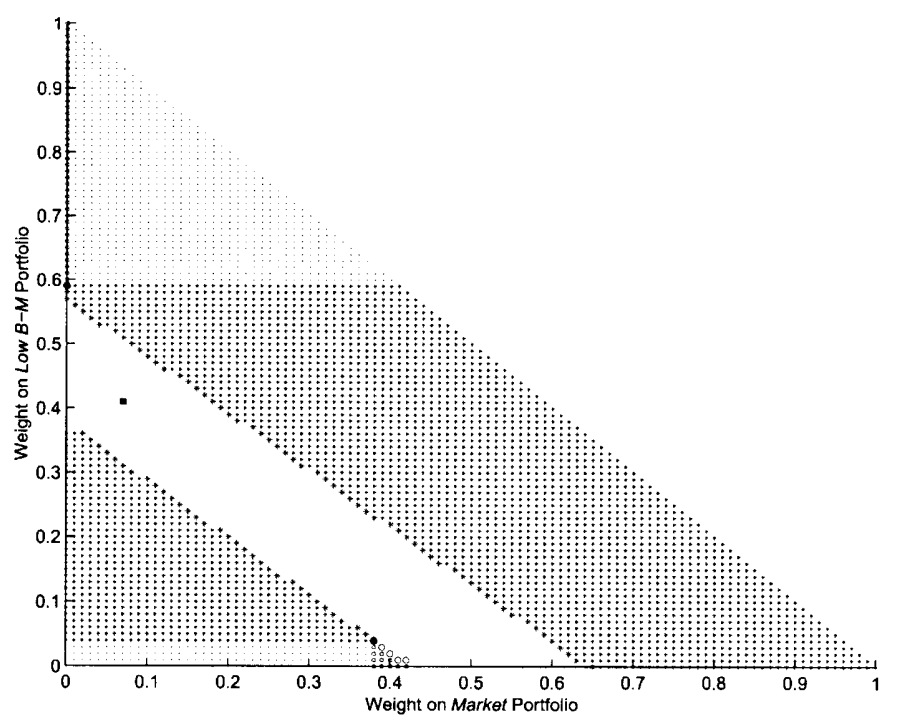

6.c dividend state $=19$

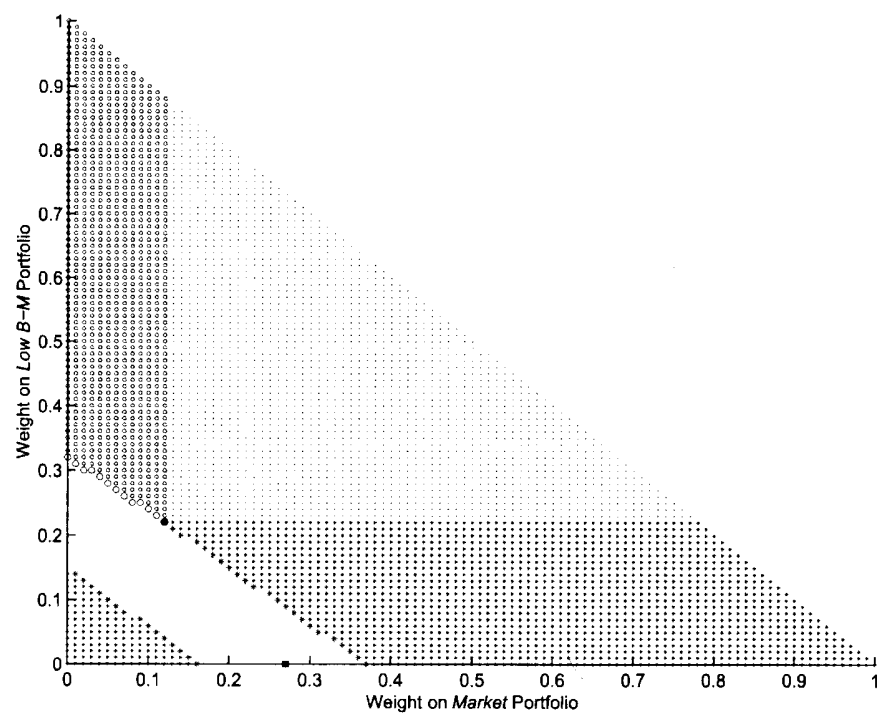

6.b dividend state $=10$

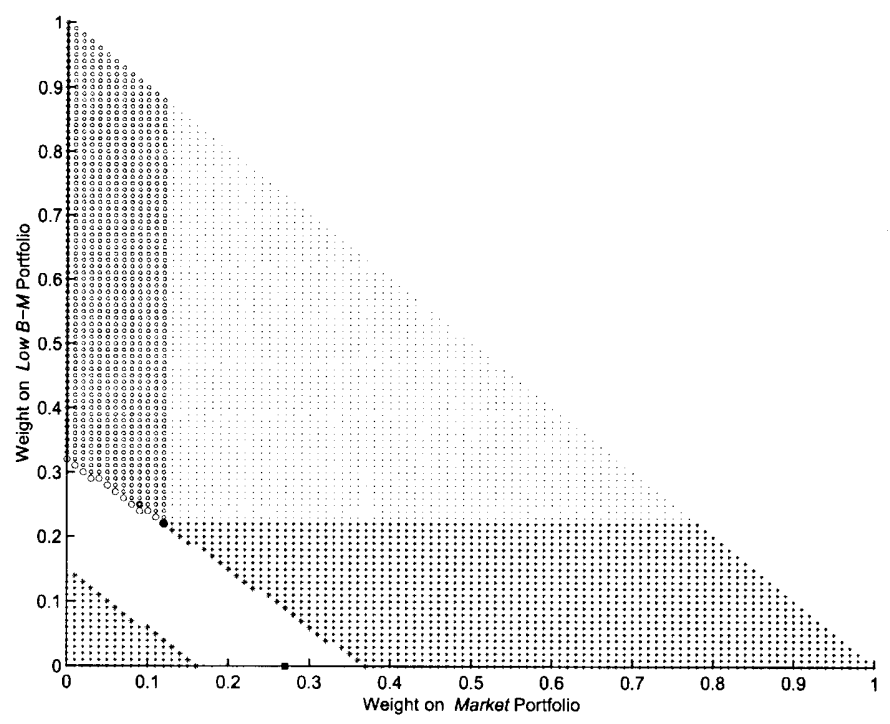

6.d i.i.d returns

Figure 6. Rebalancing rules with proportional costs \& Predictable returns: Data correlation. The two risky assets available are a portfolio of low book-to-market stocks (low B-M asset) and the market. Each blank region and its larger-marker boundary represents the allocation states in which the agent does not trade. Larger markers represent target portfolios for the states represented by the analogous smaller markers. Each allocation state marked by a smaller solid dot rebalances to the closest larger solid dot: both weights change with such rebalancing. Each allocation state marked by a smaller cross (circle) rebalances horizontally (vertically) to the corresponding larger cross (circle): only the weight on the market (low B-M asset) changes with such rebalancing. Subfigures a, b, c (e, f, g) report for the last (first) month of the 20-year investment horizon when returns are predictable. Subfigures $\mathrm{d}$ and $\mathrm{h}$ represent the solution when returns are i.i.d with the associated distribution for the last and the first month of the 20-year investment horizon respectively. Optimal rebalancing rule is dependent on the dividend yield state. In each case, the solid square represents the unique target portfolio in the associated no-transactions-costs world. The agent is a dynamic conditional optimizer. The conditional joint distribution of log returns and the dividend yield is represented by the Tauchen and Hussey (1991) discretization of the VAR introduced in section 3. The calibration is based on historical data from $7 / 27$ to $11 / 96$. The risk aversion parameter, $\gamma$, is fixed at 8 . We set proportional cost parameters $\phi_{p}^{\text {Market }}=0.25 \%$ and $\phi_{p}^{\text {Low B-M }}=0.375 \%$ and all other parameters to zero in equation (4) of the text. 


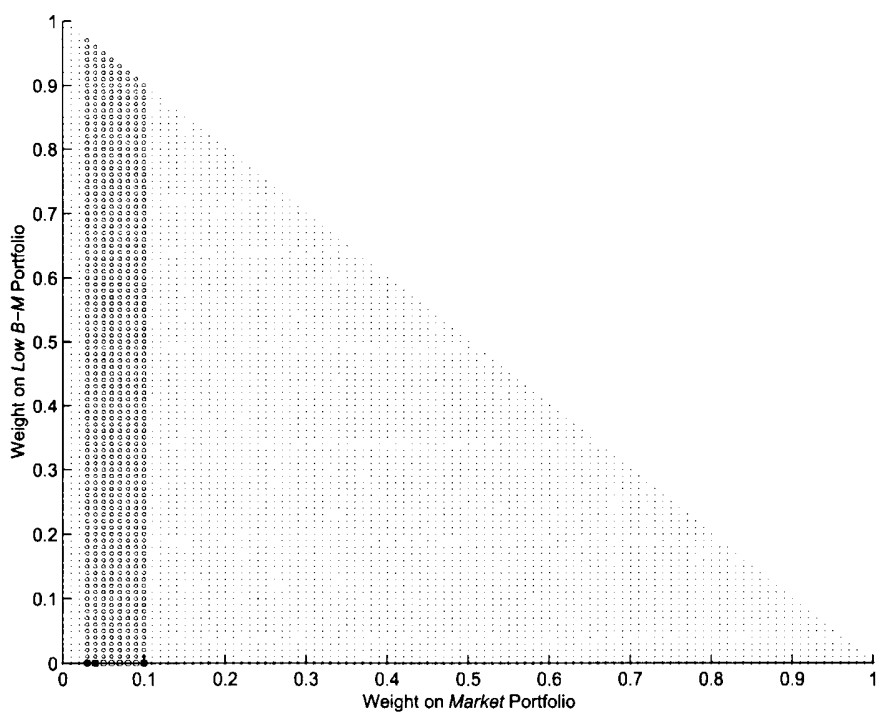

6.e dividend state $=1$

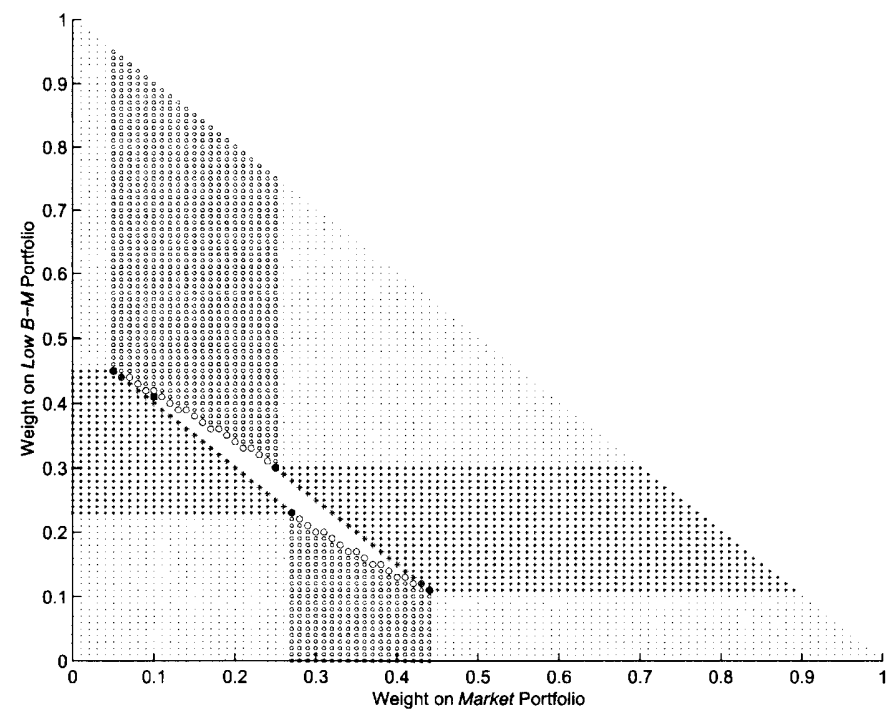

6.g dividend state $=19$

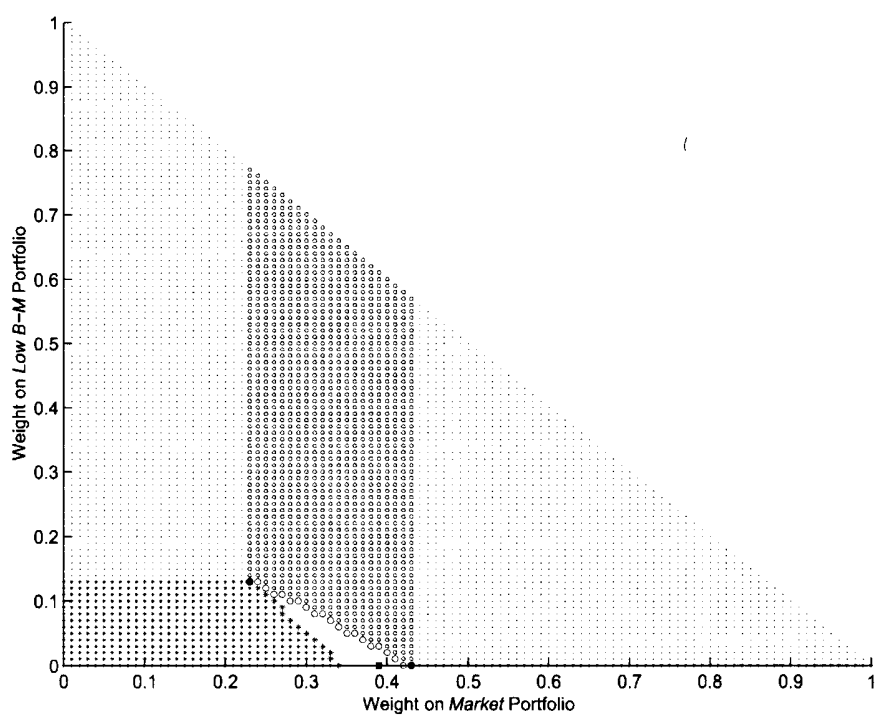

6.f $d$ ividend state $=10$

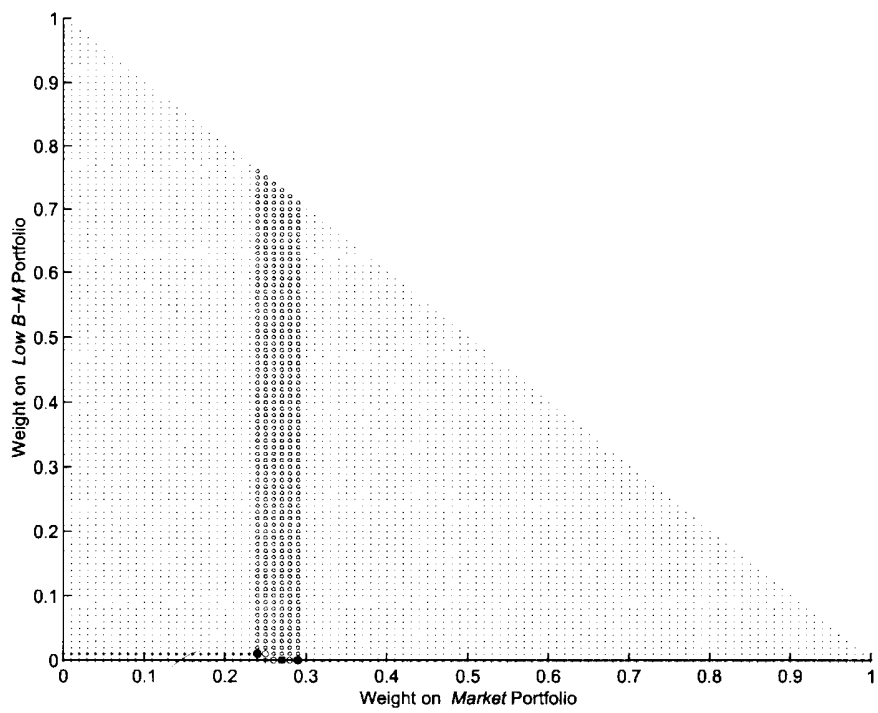

6.h $i$.i.d. returns

Figure 6. Rebalancing rules with proportional costs \& Predictable returns: Data correlation. (cont'd.) 


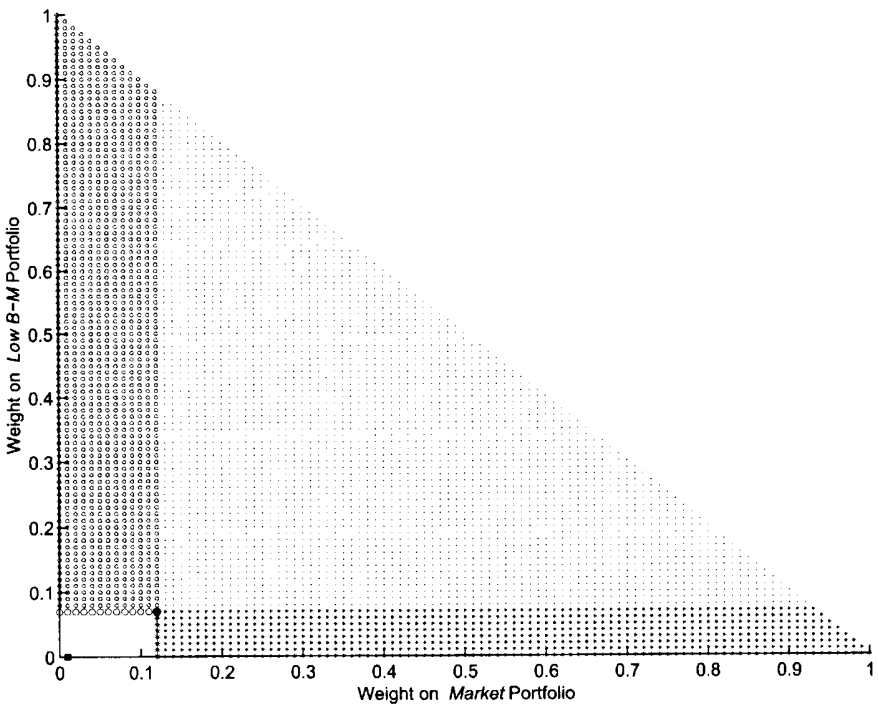

7.a dividend state $=1$

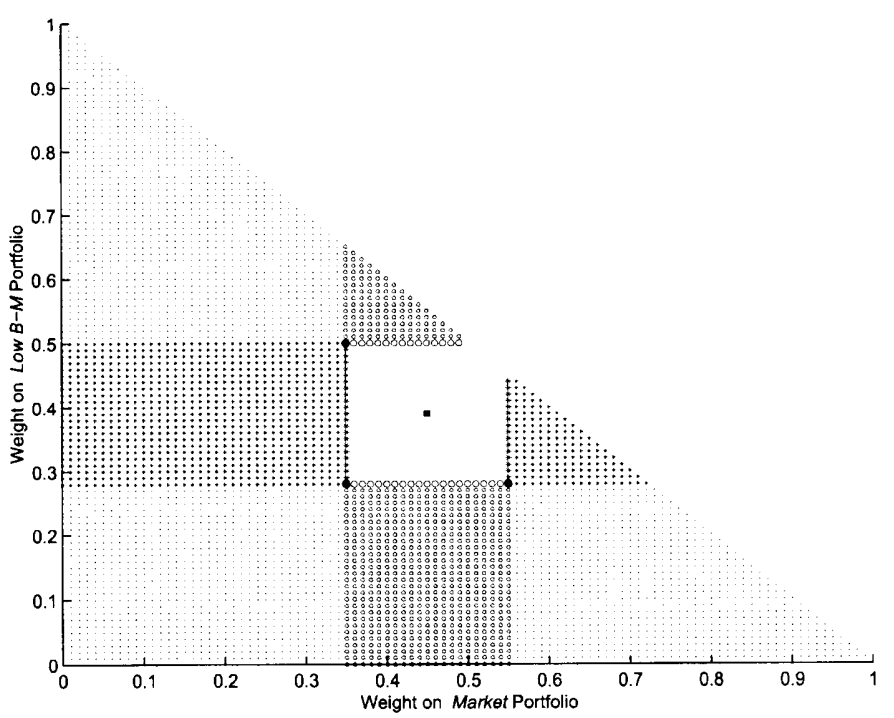

7.c $d$ ividend state $=19$

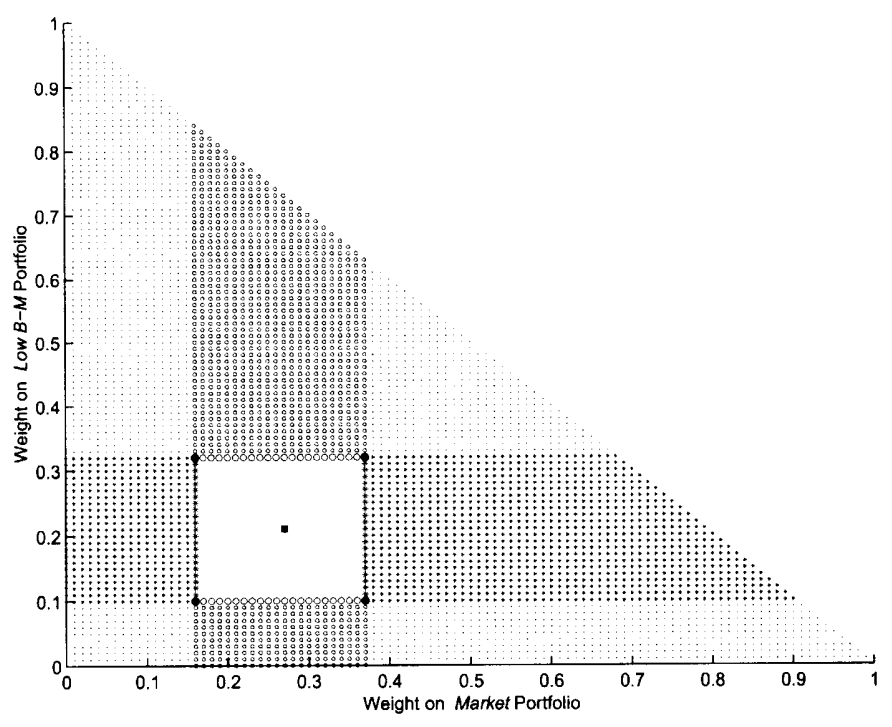

7.b dividend state $=10$

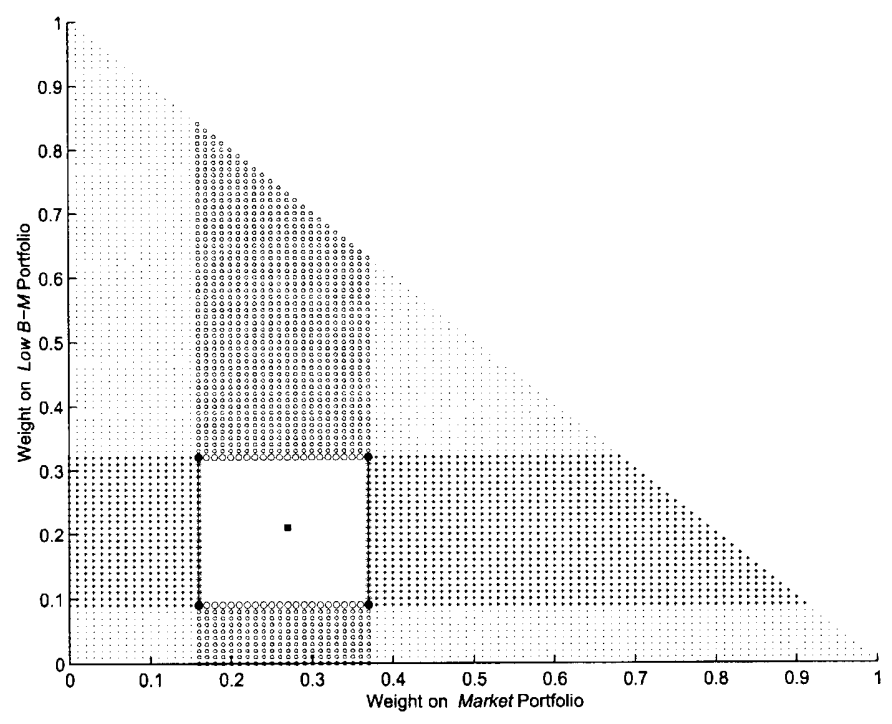

7.d i.i.d.returns

Figure 7. Rebalancing rules with proportional costs \& Predictable returns: Zero conditional correlation. The two risky assets available are a portfolio of low book-to-market stocks (low B-M asset) and the market. Each blank region and its larger-marker boundary represents the allocation states in which the agent does not trade. Larger markers represent target portfolios for the states represented by the analogous smaller markers. Each allocation state marked by a smaller solid dot rebalances to the closest larger solid dot: both weights change with such rebalancing. Each allocation state marked by a smaller cross (circle) rebalances horizontally (vertically) to the corresponding larger cross (circle): only the weight on the market (low B-M asset) changes with such rebalancing. Subfigures a, b, c (e, f, g) report for the last (first) month of the 20-year investment horizon when returns are predictable. Subfigures $d$ and $h$ represent the solution when returns are i.i.d with the associated unconditional distribution for the last and the first month of the 20-year investment horizon respectively. Optimal rebalancing rule is dependent on the dividend yield state. In each case, the solid square represents the unique target portfolio in the associated no-transactions-costs world. The agent is a dynamic conditional optimizer. The conditional joint distribution of log returns and the dividend yield is represented by the Tauchen and Hussey (1991) discretization of the VAR introduced in section 3. The calibration is based on historical data from $7 / 27$ to $11 / 96$. The risk aversion parameter, $\gamma$, is fixed at 8 . We set proportional cost parameters $\phi_{p}^{\text {Market }}=0.25 \%$ and $\phi_{p}^{\text {Low B-M }}=0.375 \%$ and all other parameters to zero in equation (4). We orthogonalize returns conditional on each dividend state. To ensue positive definiteness of the conditional covariance matrix set the conditional correlation of returns and dividend yield at $75 \%$ of the value implied by the gaussian VAR fit to the data. 


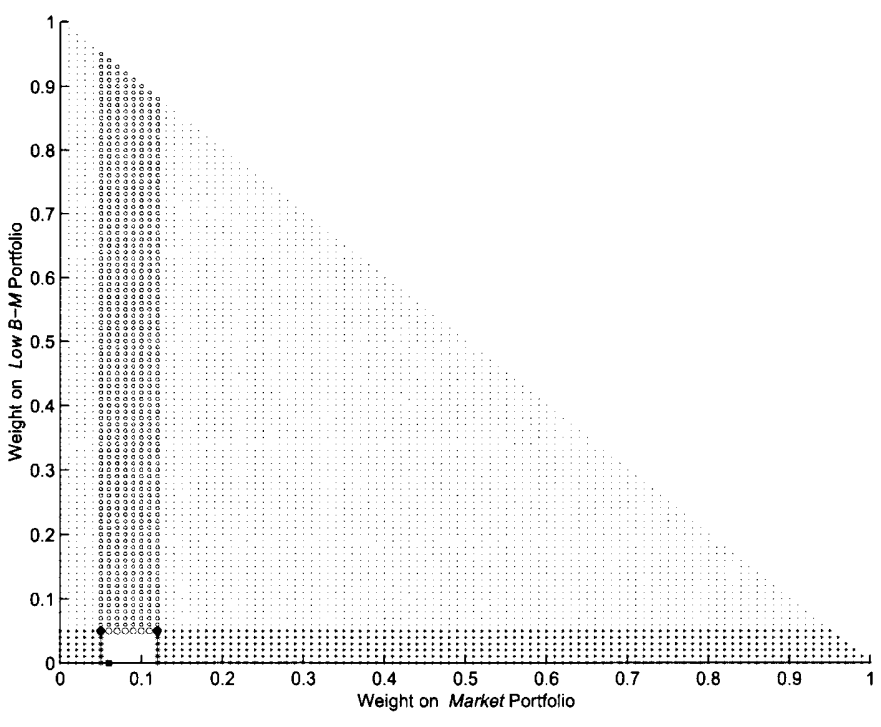

7.e dividend state $=1$

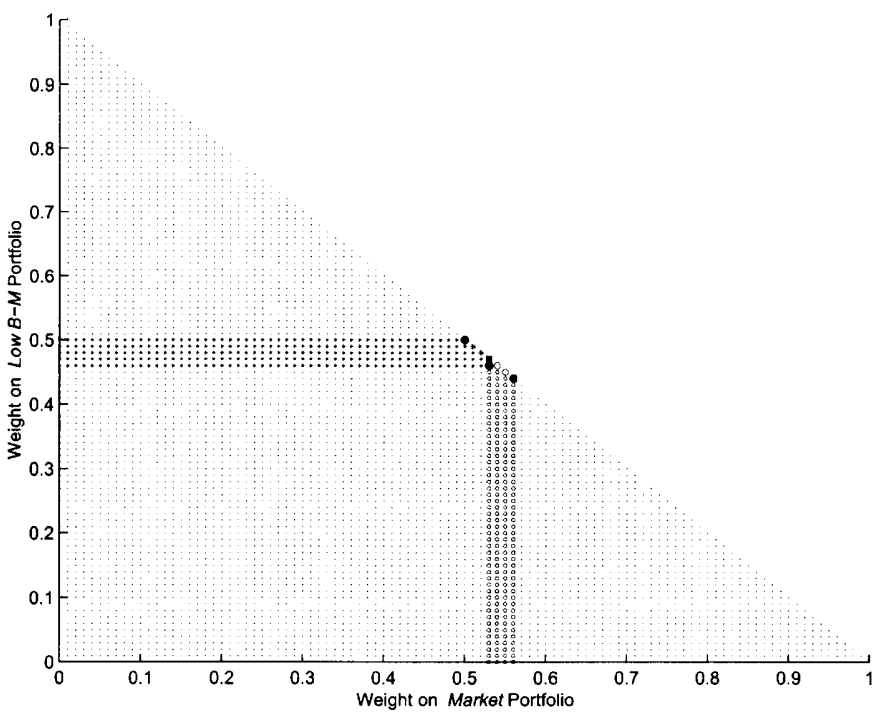

7.g dividend state $=19$

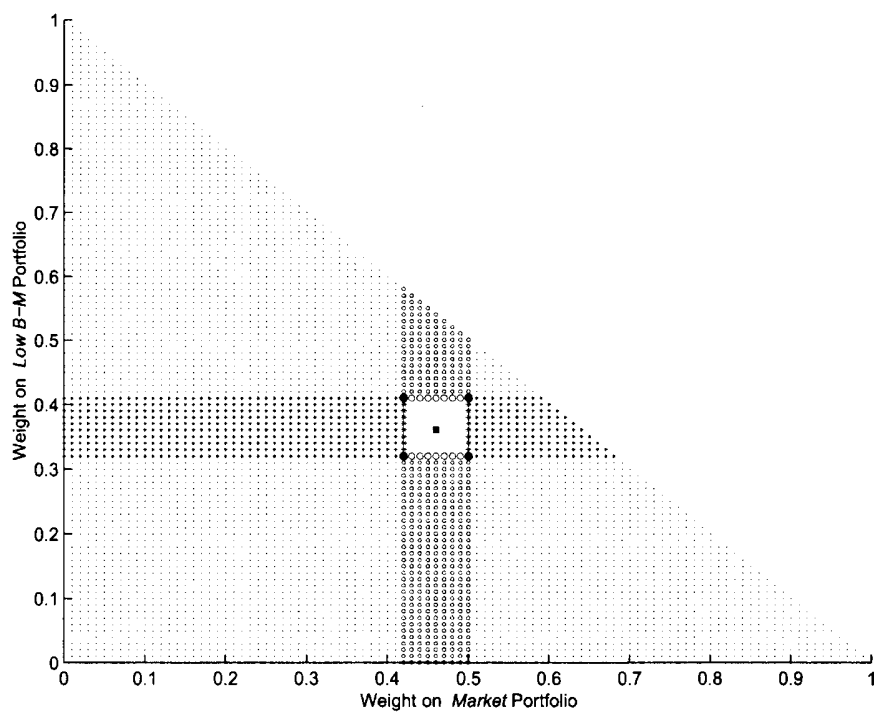

7.f dividend state $=10$

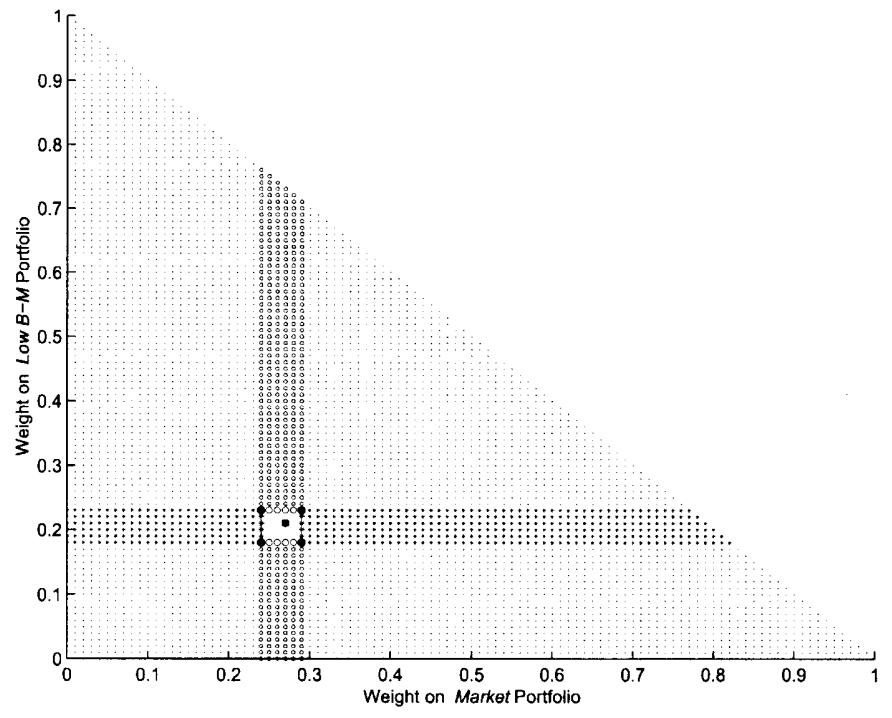

7.h i.i.d. returns

Figure 7. Rebalancing rules with proportional costs \& Predictable returns: Zero conditional correlation. (cont'd.) 


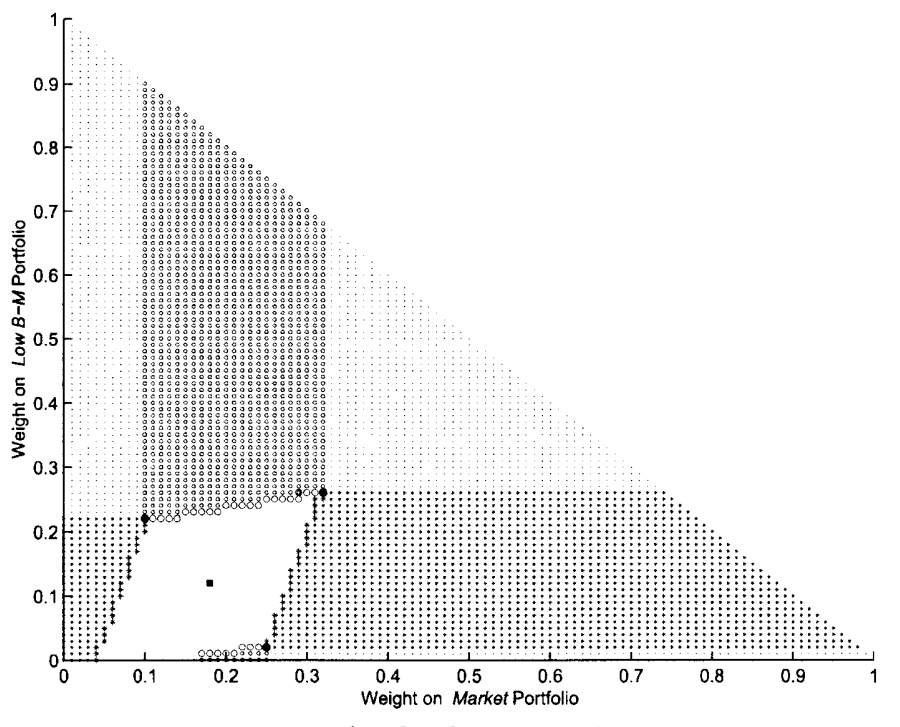

8. a dividend state $=5$

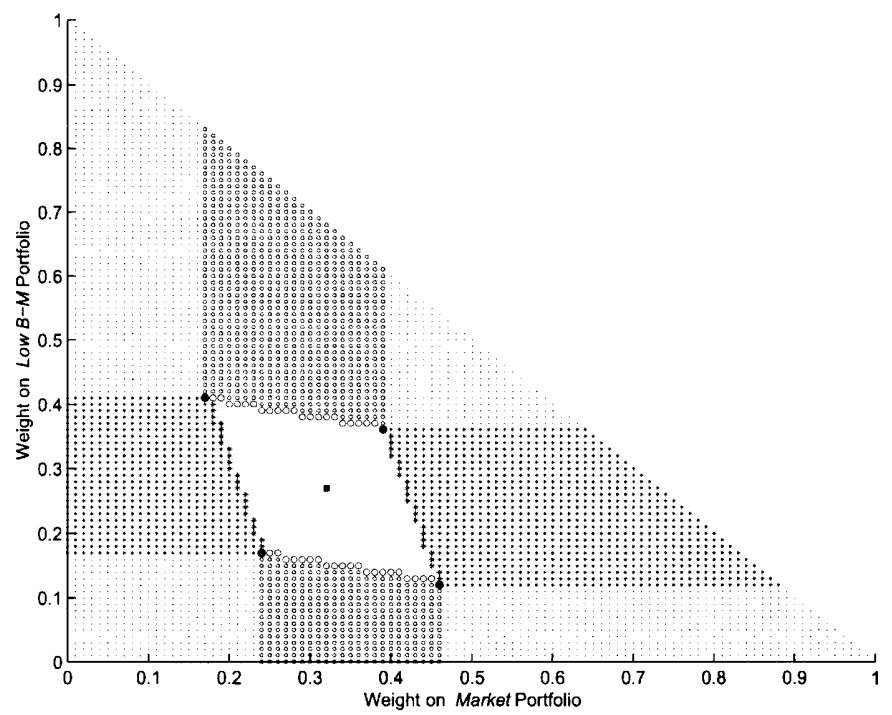

8.b dividend state $=15$

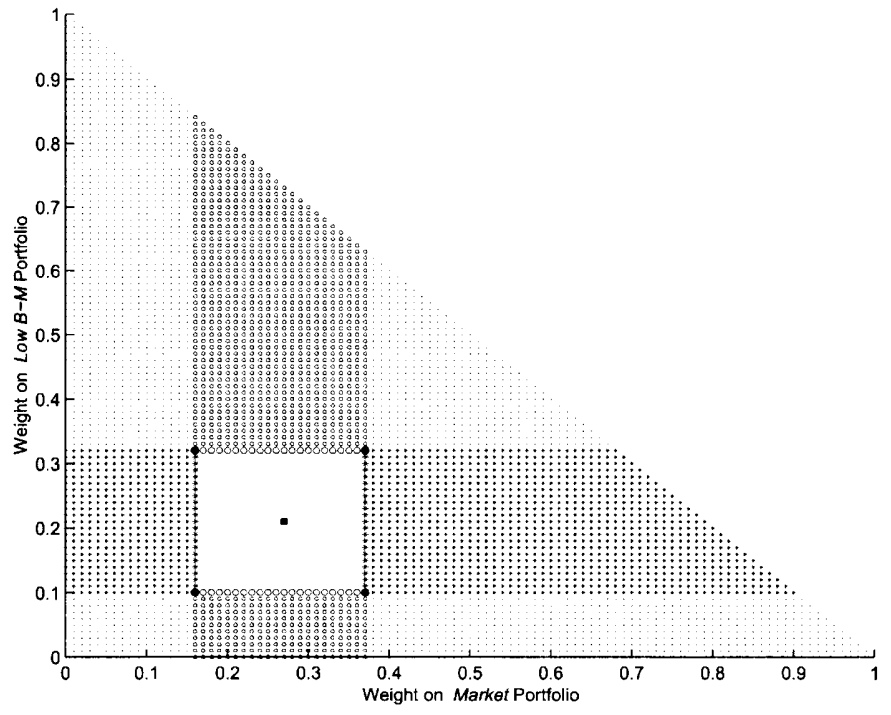

8.c dividend state $=10$

Figure 8. Rebalancing rules with proportional costs \& Predictable returns: State dependent conditional correlations. The two risky assets available are a portfolio of low book-to-market stocks (low B-M asset) and the market. Each blank region and its larger-marker boundary represents the allocation states in which the agent does not trade. Larger markers represent target portfolios for the states represented by the analogous smaller markers. Each allocation state marked by a smaller solid dot rebalances to the closest larger solid dot: both weights change with such rebalancing. Each allocation state marked by a smaller cross (circle) rebalances horizontally (vertically) to the corresponding larger cross (circle): only the weight on the market (low B-M asset) changes with such rebalancing. Subfigures a, b, c (d, e, f) report for the last (first) month of the 20-year investment horizon when returns are predictable. Optimal rebalancing rule is dependent on the dividend yield state. In each case, the solid square represents the unique target portfolio in the associated no-transactions-costs world. The agent is a dynamic conditional optimizer. Conditional joint distribution of $\log$ returns and the dividend yield is represented by a slight modification of the Tauchen and Hussey (1991) discretization of the VAR introduced in section 3. We consider a simple heteroskedastic model in which the conditional correlation between the assets is set to be positive for high dividend states (11-19), and negative for low dividend states (1-9), and zero for the middle dividend state (10). All conditional and unconditional moments of the data as implied by the homoskedastic VAR are matched except the following: unconditional correlation between the asset returns (which is set to zero in the heteroskedastic model) and the conditional and unconditional contemporaneous correlations of low B-M asset and the dividend yield. The calibration is based on historical data from $7 / 27$ to $11 / 96$. The risk aversion parameter, $\gamma$, is fixed at 8. We set proportional cost parameters $\phi_{p}^{\text {Market }}=0.25 \%$ and $\phi_{p}^{\text {Low B-M }}=0.375 \%$ and all other parameters to zero in equation (4). 


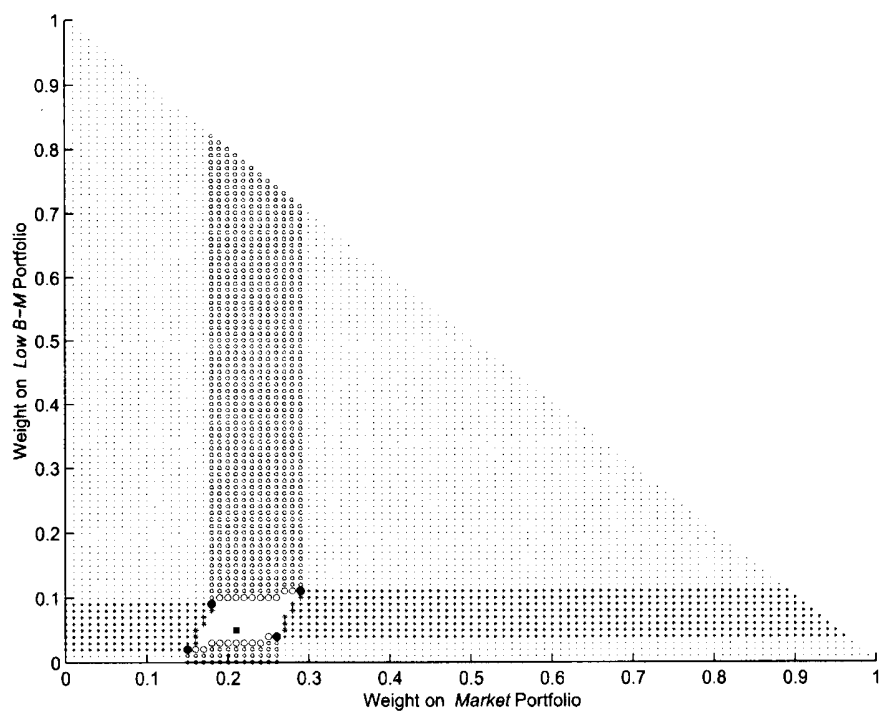

8.d dividend state $=5$

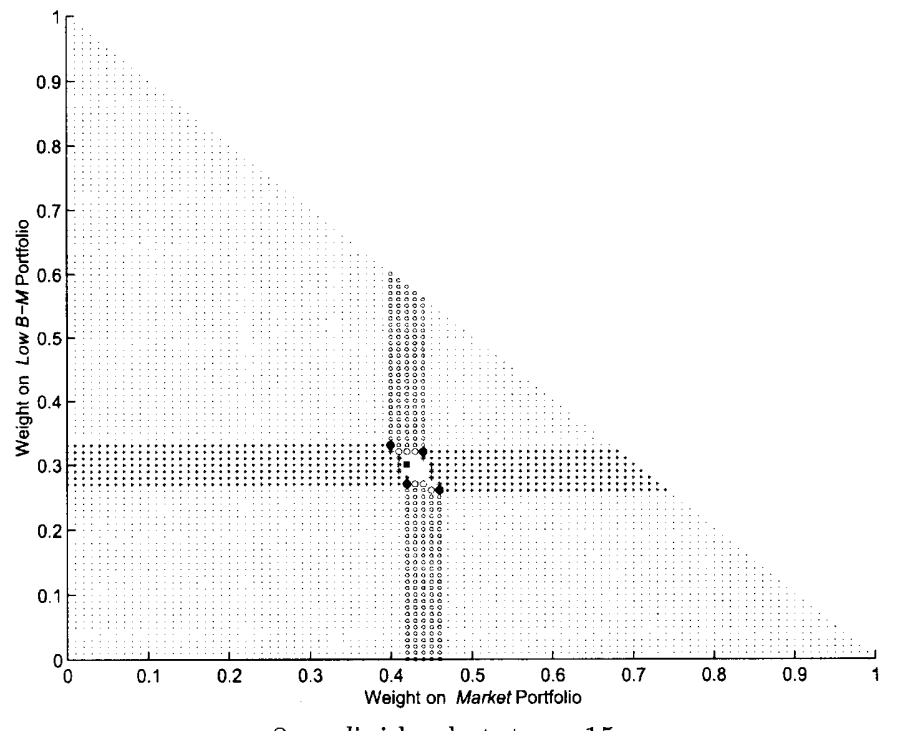

8.e dividend state $=15$

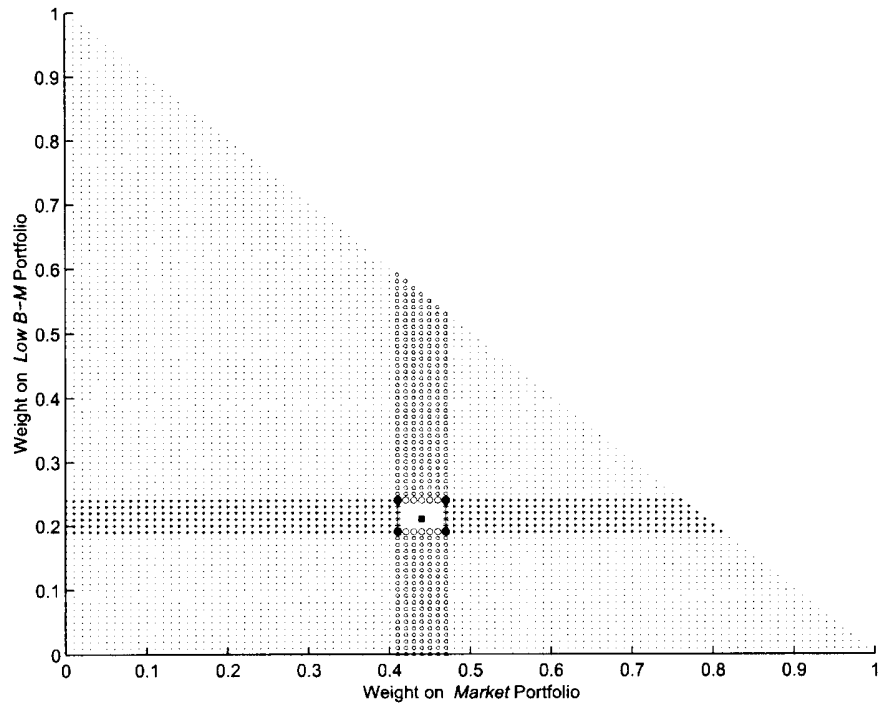

8.f dividend state $=10$

Figure 8. Rebalancing rules with proportional costs \& Predictable returns: State dependent conditional correlations. (cont'd.) 